ITEP-M6/95

OU-HET-230

\title{
PREPOTENTIAL and the SEIBERG-WITTEN THEORY
}

\author{
H.Itoyama ${ }^{1}$ and A.Morozov ${ }^{2}$
}

\begin{abstract}
Some basic facts about the prepotential in the SW/Whitham theory are presented. Consideration begins from the abstract theory of quasiclassical $\tau$-functions, which uses as input a family of complex spectral curves with a meromorphic differential $d S$, subject to the constraint $\partial d S / \partial($ moduli $)=$ holomorphic, and gives as an output a homogeneous prepotential on extended moduli space. Then reversed construction is discussed, which is straightforwardly generalizable from spectral curves to certain complex manifolds of dimension $d>1$ (like $K 3$ and $C Y$ families). Finally, examples of particular $N=2$ SUSY gauge models are considered from the point of view of this formalism. At the end we discuss similarity between the $W P_{1,1,2,2,6}^{12}$-Calabi-Yau model with $h_{21}=2$ and the 1d $S L(2)$ Calogero/Ruijsenaars model, but stop short of the claim that they belong to the same Whitham universality class beyond the conifold limit.
\end{abstract}

\footnotetext{
${ }^{1}$ Departement of Physics, Graduate School of Science, Osaka University, Toyonaka, Osaka 560, Japan. E-mail address: itoyama@funpth.phys.sci.osaka-u.ac.jp

${ }^{2}$ 117259, ITEP, Moscow, Russia. E-mail address: morozov@vxitep.itep.ru
} 


\section{Contents}

1 Introduction 3

2 Notations 4

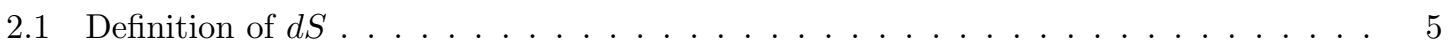

2.2 Basis in the space of solutions $\ldots \ldots \ldots \ldots \ldots \ldots \ldots$

2.3 Another basis and solutions to $\mathrm{KP} /$ Toda hierarchy $\ldots \ldots \ldots \ldots \ldots$

2.4 Relation between bases . . . . . . . . . . . . . . . . . . . . 7

$\begin{array}{lll}3 & \text { Whitham flows } & 7\end{array}$

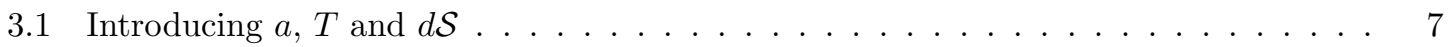

3.2 Whitham equations for $h(T) \ldots \ldots \ldots \ldots \ldots$

$3.3 \quad$ Canonical form of $d \mathcal{S} \ldots \ldots \ldots \ldots \ldots \ldots \ldots$

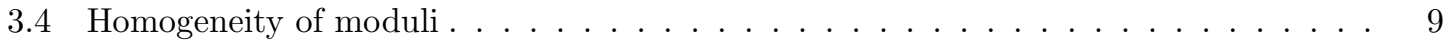

4 Prepotential. The standard case 10

4.1 Motivation . . . . . . . . . . . . . . . . . . . . . . . . 10

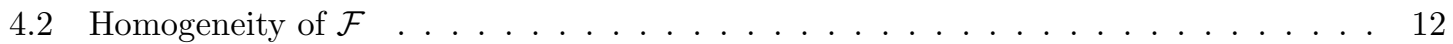

4.3 Discussion . . . . . . . . . . . . . . . . . . . . . . . . 12

4.4 Homogeneity property in terms of $d \mathcal{S} \ldots \ldots \ldots \ldots \ldots \ldots$

5 Prepotential beyond one complex dimension $\quad 14$

5.1 Notations and definition $\ldots \ldots \ldots \ldots \ldots \ldots \ldots$

5.2 Comments on requirement $(\mathrm{i}) \ldots \ldots \ldots \ldots \ldots \ldots$

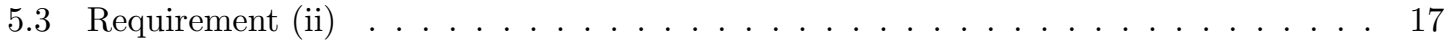

6 Examples 18

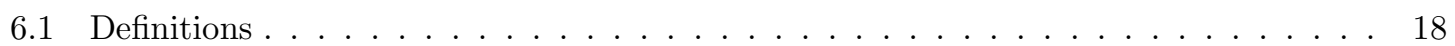

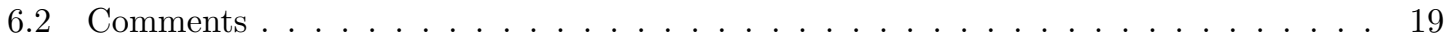

7 Examples of UV-infinite $4 d$ models: Riemann sphere as the bare spectral surface 20 
7.1 Original Seiberg-Witten (sine-Gordon) example. Curve and $d \mathcal{S}_{\min } \quad \ldots . . . . . .20$

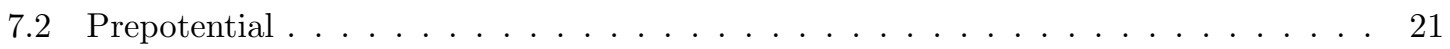

7.3 Picard-Fuchs equation . . . . . . . . . . . . . . . . . . . . 22

7.4 Toda-chain for $S L\left(N_{c}\right) \quad \ldots \ldots \ldots \ldots \ldots \ldots \ldots \ldots$

8 UV-finite $4 d$ models and the $1 d$ Calogero system: Elliptic curve as the bare spec$\begin{array}{lr}\text { tral surface } & 25\end{array}$

8.1 The case of the $S L(2)$ group. Curve, $d \mathcal{S}_{\min }$ and the prepotential $\ldots \ldots$. . . . . 25

8.2 The $m^{2}=0(N=4$ SUSY $)$ limit . . . . . . . . . . . . . . . . . . 27

8.3 The double scaling $m^{2}=\infty$ (pure gauge $N=2$ or sine-Gordon) limit . . . . . . . 28

8.4 The double scaling limit in elliptic parametrization . . . . . . . . . . . . . . . . . 29

8.5 Picard-Fuchs equations for $\oint d \mathcal{S}_{\min } \ldots \ldots \ldots \ldots \ldots$. . . . . . . . . . . . 29

8.6 The $m^{2} \rightarrow \infty, q \rightarrow 0$ double scaling $(N=2)$ limit of Picard-Fuchs equation . . . . 31

8.7 The $m^{2}=0(N=4)$ limit . . . . . . . . . . . . . . . . . . 32

8.8 Canonical form of the Picard-Fuchs equation for the case of elliptic bare spectral curve 33

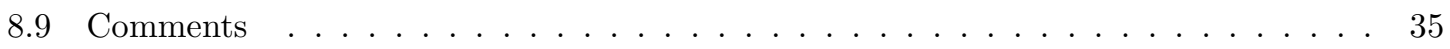

9 Calabi-Yau manifold with $h_{21}=2$ as a spectral hypersurface 36

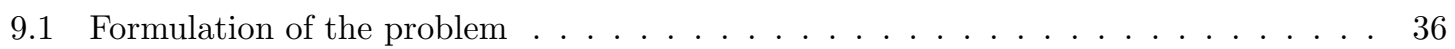

9.2 Vicinity of the conifold locus: the sine-Gordon limit of Calogero model . . . . . . . 37

9.3 Beyond conifold limit . . . . . . . . . . . . . . . . . . . . . . 38

9.4 The double scaling limit of Picard-Fuchs equation . . . . . . . . . . . . . . . 39

9.5 Comments ............................. . . 40

10 Ruijsenaars model: beginning of the story 40

11 Open questions $\quad 42$

12 Conclusion $\quad 44$

13 Acknowledgements $\quad 45$ 
According to $[1,2]$ the low-energy effective actions for $4 d N=2$ SUSY YM theories are described in terms of Riemann surfaces (complex curves), $\mathcal{C}\left(h_{k}\right)$ and certain integrals $S_{C}=\oint_{C} d S$ along noncontractable contours on $\mathcal{C}$. The simplest way to summarize these results is in terms of $1 d$ integrable systems [3]. For a brief review of this approach and references see [4]. The purpose of this paper is to present some details, concerning the adequate definition and properties of the differential $d S$. Most of them are well known to the experts, see especially $[5,6,3,7,8,9]$; our presentation is necessarily close to these references.

\section{Introduction}

There are various starting points which can serve as a motivation for the study of quasiclassical $\tau$-functions.

The most general one is as follows. Given a classical dynamical system one can think of two ways to proceed after exact angle-action variables are somehow found for it. First, one can quantize the system. Second, one can average over fast fluctuations of angle-variables and get some effective slow dynamics on the space of integrals of motion. Though seemingly different, these are exactly the same problems, at least in the first approximation (known as non-linear WKB or Bogolubov-Whitham approximation). Basically, the reason is that quantum wave functions appear from averaging along the classical trajectory - very much in the spirit of ergodicity theorems. In the modern string-theory language the classical system in question arises after some first-quantized problem is exactly solved, its effective action (generating function of all the correlators in the given background field) being a $\tau$-function of some underlying loop-group symmetry. Except for particular stringy examples, it

is yet rarely known in any explicit form. The two above mentioned problems concern deformation of classical into quantum symmetry and renormalization group flow to the low-energy (topological) field theory. Effective action arising after averaging over fast fluctuations (at the end point of RG flow) is somewhat different from original $\tau$-function, and often much simpler in some respects. Still, in varience with original one (which is a generating functional of all the matrix elements of some group), this "quasiclassical $\tau$-function" - at the present stage of our knowledge - does not have any nice group-theoretical interpretation. Moreover, there is no clear understanding what all the examples of quasiclassical $\tau$-functions, that emerge in different contexts (from application of Whitham method and from quantization procedures, from the study of KP/Toda hierarchies and that of topological theories on Calabi-Yau manifolds) have in common (see [5] and [6] for attempts of "universal" definitions). 
Emergence of quasiclassical $\tau$-functions in the role of (exponentiated) prepotentials in exactly solvable supersymmetric $4 d$ theories - though not a surprise from this general point of view unavoidably stimulates new interest to these underinvestigated objects. Moreover, their new appearance in $[1,2]$ is remarkably similar to old examples, related to $\mathrm{KP} /$ Toda hierarchies. As there, the crucial ingredient is a family of Riemann surfaces $\mathcal{C}(h)$ with meromorphic $(1,0)$-differentials $d S$, subject to the constraint that derivatives of are holomorphic. Once such family is introduced, the very existence of prepotential and its homogeneity - a subject of certain attention recently - are immediate corollaries.

It is this last relation - between the differentials $d S$ and the prepotentials - that is the topic of the present paper. We do not go into more details of above-described general philosophy [10] around the notion of $\tau$-functions - neither classical nor quasiclassical. Instead we investigate formally the corollaries of the definition of $d S$, just mentioning briefly relations to various other problems which we meet on the way. At least one of the formulations of the prepotential theory allows for immediate generalization from spectral curves to complex manifolds of arbitrary dimension - and thus identify the prepotentials of the special geometry as (logarithms of) the quasiclassical $\tau$-functions. In the last sections we go backwards, and study some particular examples, related to various $N=2$ SUSY gauge theories, showing how they suit into the general framework.

\section{Notations}

First of all, it is only cohomology class of $d S$ that matters, therefore in what follows we use "" instead of "=" to designate equivalence modulo total derivative. Futher, let $d \omega_{i}, \quad i=1, \ldots, p$ denote canonical holomorphic 1-differentials on the complex "spectral" curve (Riemann surface) $\mathcal{C}\left(h_{k}\right)$, where $p$ is the genus of $\mathcal{C}$ and $h_{k}$ are its moduli (of complex structure), so that $\oint_{A_{i}} d \omega_{j}=\delta_{i j}$ $\oint_{B_{i}} d \omega_{j}=\mathcal{T}_{i j}$, and $\mathcal{T}_{i j}\left(h_{k}\right)$ is period matrix.

From the point of view of original YM theory (refered to in this context as the "target space theory") the type of the curve $\mathcal{C}$, in particular its genus $p$, is defined by the gauge group $G$, while $h_{k}$ are the vacuum expectation values $\frac{1}{k}<\operatorname{Tr} \Phi^{k}>$ of a scalar field in the adjoint of $G$, which break original gauge symmetry down to $U(1)^{r_{G}}$. From the point of view of integrability theory, the type of $\mathcal{C}$ is related to the choice of integrable system, and $h_{k}$ are its Hamiltonians (integrals of motion). See [4] for details. 


\section{$2.1 \quad$ Definition of $d S$}

The meromorphic 1-differential $d S$ on $\mathcal{C}$ can be (partly) defined by the requirement: ${ }^{1}$

$$
\frac{\partial d S}{\partial h_{k}} \cong \text { holomorphic differential, }
$$

or, in the given basis $\left\{d \omega_{i}\right\}$,

$$
\frac{\partial d S}{\partial h_{k}} \cong \sum_{i=1}^{p} \sigma_{k i}^{(d S)} d \omega_{i} \quad \text { for all } h_{k} .
$$

Of course, everything here - $d S, \sigma, d \omega$ - depend on the moduli $h_{k}$; the coefficients $\sigma$ depend also on particular solution $d S$. The equation (2.2) is somewhat restrictive. Normally an object which is holomorphic in a given complex structure, becomes non-holomorphic in another one. At best, one can adjust things so that holomorphic objects acquire poles when differentiated with respect to the moduli. Eq.(2.2) demands quite the opposite when applied to a meromorphic $d S$ : the variation of complex structure should eliminate the poles. The option that allows meromorphic solutions to (2.2) to exist is that the newly emerging poles can coincide with the zeroes of $d S$, and thus are canceled. Another requirement is that residues of the poles of $d S$ itself, if any, should be independent of moduli.

Existence of solutions to (2.2) depend on the family of the curves $\mathcal{C}$, in particular, on the number

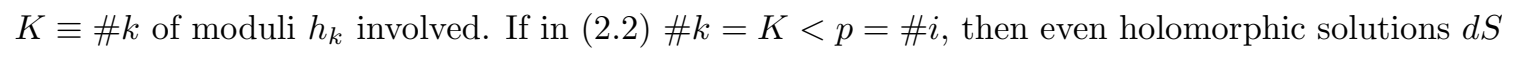
can exist. If $K=p$ - the case to be actually discussed below (and associated with pure gauge $N=2$ SUSY theories in $4 d$ and with Toda-chain systems in $1 d$ ) - there are no holomorphic solutions, but those with any non-empty set of poles do exist. If $K>p$ (as in the case of $N=2$ theory with matter multiplets in the fundamental of $G$ and also for generic families of Riemann surfaces and integrable systems), the number of poles of $d S$ is restricted from below. In what follows we concentrate on the most illuminating case of $K=p$.

\subsection{Basis in the space of solutions}

Let us introduce a basis in the linear space of solutions to (2.2). For this, as is usual in the theory of integrable systems, select two points $\xi_{ \pm}$on $\mathcal{C}$ and fix somehow a complex coordinate $\xi$ in the vicinities of $\xi_{ \pm} .{ }^{2}$ Then define $d \hat{\Omega}_{n}$ as a solution to (2.2), which satisfies additional constraints:

\footnotetext{
${ }^{1}$ This definition has motivations both in the $4 d$ and integrability theories. In the former case [1, 2] it allows one to make a positive definite Kahlerian metric on moduli space from $\operatorname{Im} \sum_{i=1}^{p} \delta \oint_{A_{i}} d S \wedge \delta \oint_{B_{i}} d S$. In the latter case it allows to build from $d S$ a solution to Whitham hierarchy.

${ }^{2}$ For notational simplicity we assume that coordinate system is common for the vicinities of $\xi_{+}$and $\xi_{-}$. Actually, this is not a restriction. Our presentation is mostly in the context of Toda-lattice hierarchy. In the case of KP,
} 
(i) $d \hat{\Omega}_{ \pm n}$ for $n \geq 1$ has a pole of order $n+1$ at $\xi_{ \pm}$and no other poles; $d \hat{\Omega}_{0}$ has simple poles at both $\xi_{+}$and $\xi_{-}$.

(ii) $d \hat{\Omega}_{ \pm n}(\xi)= \pm\left[\left(\xi-\xi_{ \pm}\right)^{-n-1}+o(1)\right] d \xi$.

$d \hat{\Omega}_{0}$ has simple poles at both $\pm \xi$ with residues \pm 1 . When $d \hat{\Omega}_{n}$ exist for all $n$ - as is the case of $K=p$, (ii) can be easily fulfilled by triangular transformation of any basis which satisfies (i). In our basis eq.(2.2) acquires the form:

$$
\frac{\partial d \hat{\Omega}_{n}}{\partial h_{k}} \cong \sum_{i=1}^{p} \sigma_{k i}^{(n)} d \omega_{i}
$$

\subsection{Another basis and solutions to KP/Toda hierarchy}

Requirements (i) and (ii) are just the same as those in the theory of KP/Toda-hierarchies [11], where instead of being a solution to $(2.2)$, the basis element $d \Omega_{n}$ is requested to satisfy:

(iii) $\oint_{A_{j}} d \Omega_{n}=0 \quad$ for all $A$-cycles.

Then, the $B$-periods of such $d \Omega_{n}, \quad \kappa_{j}^{(n)} \equiv \oint_{B_{j}} d \Omega_{n}$, are the frequencies, which - together with the twists made from $\oint_{B_{j}} d \omega_{i}=\mathcal{T}_{i j}$, enter the argument of the theta-function in the celebrated formula,

$$
\begin{gathered}
\tau(t \mid h)=e^{f\left(\alpha_{i}, t_{n} \mid h\right)} \Theta\left(s_{B_{j}} \mid \mathcal{T}_{i j}(h)\right), \\
s_{B_{j}}(\alpha, t \mid h)=\oint_{B_{j}} d s=\oint_{B_{j}}\left(\sum_{i=1}^{p} \alpha_{i} d \omega_{i}+\sum_{n=-\infty}^{\infty} t_{n} d \Omega_{n}\right)=\sum_{i=1}^{p} \alpha_{i} \mathcal{T}_{i j}+\sum_{n=-\infty}^{\infty} \kappa_{j}^{(n)}(h) t_{n},
\end{gathered}
$$

for $\mathrm{KP} /$ Toda $\tau$-function, associated with the Riemann surface $\mathcal{C}$ (a $p$-zone solution). The main theorem of the $\mathrm{KP} /$ Toda theory [11] says that $h_{k}$ in eq.(2.4) are independent of $\alpha_{i}, t_{n}$ (moduli are invariants of $\mathrm{KP} /$ Toda flows). Thus $s_{B_{j}}$ are linear functions of $\alpha_{i}$ and $t_{n}$ :

$$
\mathcal{T}_{i j}=\frac{\partial s_{B_{j}}}{\partial \alpha_{i}}, \quad \kappa_{j}^{(n)}=\frac{\partial s_{B_{j}}}{\partial t_{n}}, \quad \text { and } \quad \frac{\partial \mathcal{T}_{i j}}{\partial \alpha_{i^{\prime}}}=0, \quad \frac{\partial \kappa_{j}^{(n)}(h)}{\partial t_{n}}=0 .
$$

One can also introduce $s_{A_{j}}=\oint_{A_{j}} d s=\alpha_{j}$. These are of course integrals of $t$-flows, in the sense that $\frac{\partial s_{A_{j}}}{\partial t_{n}}=0$, while $\frac{\partial s_{A_{j}}}{\partial \alpha_{i}}=\oint_{A_{j}} d \omega_{i}=\delta_{i j}$. Exponent $f\left(\alpha_{i}, t_{n} \mid h\right)$ in (2.4) is certain second-order polinomial in $\alpha_{i}$ and $t_{n}$, though a non-trivial function of the moduli $h$.

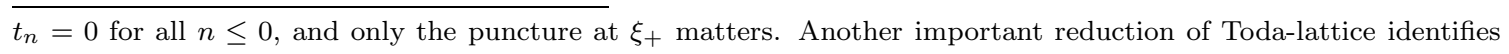
$t_{-n}=(-)^{n-1} t_{n}$, what can be also described as substitution of the curve $\mathcal{C}$ by $\hat{\mathcal{C}}$ of which $\mathcal{C}$ is a double cover. In coordinates this can be described as $\frac{1}{\xi-\xi_{0}}=\frac{1}{\xi-\xi_{+}}-\frac{1}{\xi-\xi_{-}}$(the most familiar formula arises for $\xi_{+}=0, \xi_{-} \xi_{0}=\infty$ : $\left.\hat{\xi}=\xi+\xi^{-1}\right)$. Instead of reductions of Toda-lattice one can instead consider generalizations, when there are many punctures at points $\xi_{\alpha}$. Associated time-variables are $T_{\alpha, n}, n>0$, together with certain set of zero-time variables. Additional punctures appear in considerations of $N=2$ SUSY theories with additional matter supermultiplets. 
Given (i) and (ii), the frequencies $\kappa_{j}^{(n)}$ can be also represented as residues: ${ }^{3}$

$$
\kappa_{j}^{( \pm n)}=\oint_{B_{j}} d \Omega_{ \pm n}=\frac{1}{n} \oint_{\xi_{ \pm}}\left(\xi-\xi_{ \pm}\right)^{-n} d \omega_{j}(\xi)=\frac{2 \pi i}{n} \operatorname{res}_{\xi_{ \pm}}\left(\xi-\xi_{ \pm}\right)^{-n} d \omega_{j},
$$

so that

$$
s_{B_{j}}=\oint_{B_{j}}\left(\sum_{i=1}^{p} \alpha_{i} d \omega_{i}\right)+2 \pi i \cdot \operatorname{res}\left(\sum_{n>0} \frac{t_{n}}{n}\left(\xi-\xi_{+}\right)^{-n}+\sum_{n>0} \frac{t_{-n}}{n}\left(\xi-\xi_{-}\right)^{-n}+t_{0} \log \frac{\xi-\xi_{+}}{\xi-\xi_{-}}\right) d \omega_{j} .
$$

\subsection{Relation between bases}

The basis elements $d \hat{\Omega}_{n}$ and $d \Omega_{n}$ are related, the difference between them being somehow expressed through holomorphic differentials (just because (i) and (ii) define $d \Omega_{n}$ modulo any combination of $d \omega_{i}$ - which is then fixed in a different ways either by (2.3) or by (iii)):

$$
d \hat{\Omega}_{n} \cong d \Omega_{n}+\sum_{i=1}^{p} c_{i}^{(n)} d \omega_{i}
$$

Clearly, coefficients are given by

$$
c_{i}^{(n)}(h)=\oint_{A_{i}} d \hat{\Omega}_{n}
$$

\section{Whitham flows}

\subsection{Introducing $a, T$ and $d \mathcal{S}$}

As a first step towards construction of a "quasiclassical $\tau$-function" - of which the SW prepotential is an example - let us introduce a solution $d \mathcal{S}\left(T_{n} \mid h_{k}\right)$ of (2.2), depending on (infinitely many) "slow" time-variables $T_{n}$, such that

$$
\frac{\partial d \mathcal{S}}{\partial T_{n}} \cong d \Omega_{n}
$$

Essentially, $d \mathcal{S}\left(T_{n} \mid h_{k}\right)$ is a kind of generating function for all the solutions of (2.2). Nowadays, the special notation is used for the periods $\mathcal{S}_{C} \equiv \oint_{C} d \mathcal{S}$ (motivated by relation to the $d=4$ gauge theory):

$$
a_{j} \equiv \oint_{A_{j}} d \mathcal{S}, \quad a_{j}^{D} \equiv \oint_{B_{j}} d \mathcal{S}
$$

3 This follows from the identity $d \Omega_{n} \wedge d \omega_{j}=0$ after integration over entire Riemann surface $\mathcal{C}$ :

$$
0=\int_{\mathcal{C}} d \Omega_{n} \wedge d \omega_{j}=\int_{\mathcal{C}} d\left(\Omega_{n} d \omega_{j}\right)=\sum_{i=1}^{p}\left(\oint_{A_{i}} d \Omega_{n} \oint_{B_{i}} d \omega_{j}-\oint_{B_{i}} d \Omega_{n} \oint_{A_{i}} d \omega_{j}\right)+2 \pi i \cdot \operatorname{res}_{\xi_{ \pm}}\left[\Omega_{n} d \omega_{j}\right],
$$

together with the asymptotical convention (ii). 
and we have:

$$
\frac{\partial a_{j}}{\partial T_{n}}=0, \quad \frac{\partial a_{j}^{D}}{\partial T_{n}}=\kappa_{j}^{(n)}(h)
$$

\subsection{Whitham equations for $h(T)$}

Being solution to $(2.2), d \mathcal{S}$ can be expanded in the linear basis $\left\{d \hat{\Omega}_{n}\right\}$ :

$$
d \mathcal{S} \cong \sum_{m=-\infty}^{\infty} u_{m}(T) d \hat{\Omega}_{m}(h)
$$

where coefficients $u_{m}(T)$ are independent of $h$. Substituting (3.4) into (3.1), we obtain:

$$
\begin{aligned}
& \frac{\partial d \mathcal{S}}{\partial T_{n}} \cong \sum_{m=-\infty}^{\infty}\left(\frac{\partial u_{m}}{\partial T_{n}} d \hat{\Omega}_{m}+u_{m} \frac{\partial d \hat{\Omega}_{m}}{\partial h_{k}} \frac{\partial h_{k}}{\partial T_{n}}\right) \stackrel{(2.3)}{\cong} \\
& \cong \sum_{m=-\infty}^{\infty}\left(\frac{\partial u_{m}}{\partial T_{n}} d \hat{\Omega}_{m}+u_{m} \sum_{k} \frac{\partial h_{k}}{\partial T_{n}} \sum_{i=1}^{p} \sigma_{k i}^{(m)}(h) d \omega_{i}\right) .
\end{aligned}
$$

On the other hand, $\frac{\partial d \mathcal{S}}{\partial T_{n}} \cong d \Omega_{n} \cong d \hat{\Omega}_{n}-\sum_{i=1}^{p} c_{i}^{(n)} d \omega_{i}$, and from comparison of these expressions we derive:

$$
\frac{\partial u_{m}}{\partial T_{n}}=\delta_{m n}, \text { i.e. } u_{m}(T)=T_{m},
$$

along with the "Whitham equations",

$$
\sum_{k} \frac{\partial h_{k}}{\partial T_{n}}\left(\sum_{m=-\infty}^{\infty} T_{m} \sigma_{k i}^{(m)}\right) \equiv \sum_{k} \frac{\partial h_{k}}{\partial T_{n}} \sigma_{k i}=-c_{i}^{(n)} .
$$

Condensed notation $\sigma_{k i} \equiv \sum_{m=-\infty}^{\infty} T_{m} \sigma_{k i}^{(m)}$ will be of use in the further manipulations.

Thus we see that the moduli $h_{k}$ that were integrals of $t$-flows, are unavoidably $T$-dependent.

\subsection{Canonical form of $d \mathcal{S}$}

Equation (3.6) implies now that

$$
d \mathcal{S} \cong \sum_{m=-\infty}^{\infty} T_{m} d \hat{\Omega}_{m}(h \mid T) \stackrel{(2.6)}{\cong} \sum_{m=-\infty}^{\infty}\left(T_{m} d \Omega_{m}+T_{m} \sum_{i=1}^{p} c_{i}^{(m)} d \omega_{i}\right) .
$$

Integrating this along $A_{j}$-cycles and using (2.7) we get:

$$
a_{j}=\oint_{A_{j}} d \mathcal{S}=\sum_{m=-\infty}^{\infty} T_{m} c_{j}^{(m)}=\sum_{m=-\infty}^{\infty} T_{m} \oint_{A_{j}} d \hat{\Omega}_{m},
$$

and substituting this back into (3.8),

$$
d \mathcal{S} \cong \sum_{i=1}^{\infty} a_{i} d \omega_{i}+\sum_{m=-\infty}^{\infty} T_{m} d \Omega_{m}
$$


Note that, despite $a_{i}=a_{i}(h(T))$ depend on $h_{k}$, which are $T$-dependent, this dependence cancels completely in $a_{i}\left(h_{k}\right)$ - in accordance with (3.3). One can say also that solution to Whitham differential equations (3.7) contain integration constants - and these are precisely the $a_{i}(h)$. This implies that one can add $a_{i}$ as extra independent variables to the set of "slow" times $T$, and $d \mathcal{S}=d \mathcal{S}\left(a_{i}, T_{n}\right)$.

Once this is done, (3.1) should be supplemented by an expression for $\frac{\partial d \mathcal{S}}{\partial a_{i}}$. However, it can not be postulated arbitrarily, since we already defined $d \mathcal{S}$ unambigously: this expression should be derived. It is at this point that the restriction $K=p$ is used for the first time. Let us consider a variation of (3.9):

$$
\delta a_{j}=\sum_{m=-\infty}^{\infty}\left(\delta T_{m} \oint_{A_{j}} d \hat{\Omega}_{m}+T_{m} \sum_{k} \delta h_{k} \oint_{A_{j}} \frac{\partial d \hat{\Omega}_{m}}{\partial h_{k}}\right)=\sum_{k} \delta h_{k} \sigma_{k j}+\sum_{m=-\infty}^{\infty} \delta T_{m} c_{j}^{(m)} .
$$

When $\# k=K=p=\# i, \quad \sigma_{k j}$ is a square matrix and can be inverted. Thus, puting $\delta T_{m}=0$ in (3.11), we get:

$$
\left.\frac{\partial h_{k}}{\partial a_{j}}\right|_{T_{n}=\text { const }}=\sigma_{j k}^{-1}(h)
$$

Consequently,

$$
\begin{gathered}
\left.\frac{\partial d \mathcal{S}}{\partial a_{j}}\right|_{T_{n}=\text { const }} \cong \sum_{m=-\infty}^{\infty} T_{m} \frac{\partial d \hat{\Omega}_{m}}{\partial a_{j}} \cong \sum_{m=-\infty}^{\infty} T_{m} \sum_{k} \frac{\partial h_{k}}{\partial a_{j}} \frac{\partial d \hat{\Omega}_{m}}{\partial h_{k}} \cong \\
\cong \sum_{k} \sigma_{j k}^{-1} \sum_{m=-\infty}^{\infty} T_{m} \sigma_{k i}^{(m)} d \omega_{i}=\sum_{k} \sigma_{j k}^{-1} \sigma_{k i} d \omega_{i}=d \omega_{j} .
\end{gathered}
$$

To summarize,

$$
\begin{aligned}
& d \mathcal{S} \cong \sum_{m=-\infty}^{\infty} T_{m} d \hat{\Omega}_{m} \cong \sum_{i=1}^{p} a_{i} d \omega_{i}+\sum_{m=-\infty}^{\infty} T_{m} d \Omega_{m}, \\
& \frac{\partial d \mathcal{S}}{\partial a_{i}} \stackrel{K=p}{\cong} d \omega_{i}, \quad \frac{\partial d \mathcal{S}}{\partial T_{n}} \cong d \Omega_{n} .
\end{aligned}
$$

We emphasize that these simple formulas hold, despite the fact that $a_{i}, d \omega_{i}$ and $d \Omega_{n}$ depend (only) on the moduli $h_{k}$, which in turn depend non-trivially on T's: $h_{k}(T)$. Moreover, after $a_{i}$ 's are introduced as independent variables, and whenever eq.(3.12) can make sense (e.g. if $K=p$ ), we rather write $h_{k}\left(a_{i}, T_{n}\right)$ instead of $a_{i}\left(h_{k}(T)\right)$.

\subsection{Homogeneity of moduli}

The functions $h_{k}\left(a_{i}, T_{n}\right)$, though quite complicated, are always homogeneous:

$$
\left(\sum_{i=1}^{p} a_{i} \frac{\partial}{\partial a_{i}}+\sum_{n=0}^{\infty} T_{n} \frac{\partial}{\partial T_{n}}\right) h_{k}=0 .
$$


This is a simple corollary of (3.11), which can be rewritten as

$$
\delta h_{k}=\sum_{j=1}^{p}\left(\delta a_{j}-\sum_{m=-\infty}^{\infty} c_{j}^{(m)} \delta T_{m}\right) \sigma_{j k}^{-1} .
$$

For specific (scale) variation $\delta a_{j}=\varepsilon a_{j}, \delta T_{n}=\varepsilon T_{n}$, we get:

$$
\delta_{\varepsilon} h_{k}=\varepsilon \sum_{j=1}^{p}\left(a_{j}-\sum_{m=-\infty}^{\infty} c_{j}^{(m)} T_{m}\right) \sigma_{j k}^{-1} \stackrel{(3.9)}{=} 0
$$

as stated in (3.15). This homogeneity condition is of crucial importance for the possibility to introduce prepotential in any explicit form.

\section{Prepotential. The standard case}

\subsection{Motivation}

Let us turn now to the $B$-periods of $d \mathcal{S}$ :

$$
a_{j}^{D}=\oint_{B_{j}} d \mathcal{S}=\oint_{B_{j}}\left(\sum_{i=1}^{p} a_{i} d \omega_{i}+\sum_{n=-\infty}^{\infty} T_{n} d \Omega_{n}\right)=\sum_{i=1}^{p} a_{i} \mathcal{T}_{i j}(h)+\sum_{n=-\infty}^{\infty} T_{n} \kappa_{j}^{(n)}(h) .
$$

Further, evaluate

$$
\frac{\partial a_{j}^{D}}{\partial a_{i}}=\oint_{B_{j}} \frac{\partial d \mathcal{S}}{\partial a_{i}}=\oint_{B_{j}} d \omega_{i}=\mathcal{T}_{i j}(h)
$$

Since the period matrix is symmetric, $\mathcal{T}_{i j}=\mathcal{T}_{j i}$, this implies that

$$
a_{j}^{D}=\frac{\partial \mathcal{F}}{\partial a_{j}}
$$

for some function $\mathcal{F}\left(a_{i}, T_{n}\right)$ often called a (logarithm of a) "quasiclassical $\tau$-function" or, simply, a prepotential. The arguments of this function are essentially the moduli of solutions to original (KP/Toda-like or low-energy Yang-Mills) dynamical system, with $a_{i}\left(h_{k}\right)$ parametrizing the moduli of the curve $\mathcal{C}$ and $T_{n}$ - those of coordinate systems in the vicinity of the punctures.

If $\mathcal{F}$ was non-singular when all the times $T_{n}=0$, one could use the homogeneity property (3.15) to write down the "reduced" prepotential $\left.\hat{\mathcal{F}}_{\text {red }}\left(a_{i}\right) \equiv \hat{\mathcal{F}}\left(a_{i}, T_{n}\right)\right|_{\text {all } T_{n}=0}$ in a rather explicit form:

$$
\hat{\mathcal{F}}_{\text {red }}\left(a_{i}\right)=\frac{1}{2} \sum_{i, j=1}^{p} a_{i} a_{j} \mathcal{T}_{i j}(h) .
$$

For a proof, note first of all that this is not a trivial formula, since $h$ depend non-triviall on $a_{i}$ 's. However, $h_{k}\left(a_{i}\right)$ are homogeneous of degree zero, thus the same is true about $\mathcal{T}_{i j}(h(a))$, and 
consequently $\hat{\mathcal{F}}_{\text {red }}$ is homogeneous of degree 2 :

$$
\begin{gathered}
\hat{\mathcal{F}}_{\text {red }}=\frac{1}{2} \sum_{i=1}^{p} a_{i} \frac{\partial \hat{\mathcal{F}}_{\text {red }}}{\partial a_{i}}= \\
=\frac{1}{2} \sum_{i=1}^{p} a_{i} a_{i} \stackrel{(3.2)}{=} \frac{1}{2} \sum_{i=1}^{p} a_{i} \oint_{B_{i}} d \mathcal{S} \stackrel{(3.14)}{=} \frac{1}{2} \sum_{i, j=1}^{p} a_{i} a_{j} \mathcal{T}_{i j}(h) .
\end{gathered}
$$

Unfortunately, the point $\left\{\right.$ all $\left.T_{n}=0\right\}$ is usually singular, ${ }^{4} \hat{\mathcal{F}}_{\text {red }}$ is not defined and this simple reasoning does not work: the $T$-dependence of $\mathcal{F}$ should be taken into account.

Though we did not yet define $\frac{\partial \mathcal{F}}{\partial T_{n}}$ explicitly, there is not too much freedom left. Indeed, from eq.(4.3)

$$
\frac{\partial}{\partial a_{i}}\left(\frac{\partial \mathcal{F}}{\partial T_{ \pm n}}\right)=\frac{\partial^{2} \mathcal{F}}{\partial a_{i} \partial T_{ \pm n}} \stackrel{(4.3)}{=} \frac{\partial a_{i}^{D}}{\partial T_{ \pm n}} \stackrel{(3.3)}{=} \kappa_{i}^{( \pm n)} \stackrel{(2.5)}{=} \frac{1}{n} \oint_{\xi_{ \pm}}\left(\xi-\xi_{ \pm}\right)^{-n} d \omega_{i}(\xi)
$$

Since from $(3.14) d \omega_{i} \cong \frac{\partial \mathcal{S}}{\partial a_{i}}$, we conclude that

$$
\begin{gathered}
\frac{\partial \mathcal{F}}{\partial T_{ \pm n}}=\frac{1}{n} \oint_{\xi_{ \pm}}\left(\xi-\xi_{ \pm}\right)^{-n} d \mathcal{S}(\xi), \quad n \geq 1 \\
\frac{\partial \mathcal{F}}{\partial T_{0}}=\oint_{\text {around the cut }} \log \frac{\xi-\xi_{+}}{\xi-\xi_{-}} d \mathcal{S}(\xi)=2 \pi \int_{\xi_{-}}^{\xi_{+}} d \mathcal{S}(\xi) .
\end{gathered}
$$

This should be added to (4.3),

$$
\frac{\partial \mathcal{F}}{\partial a_{i}}=\oint_{B_{i}} d \mathcal{S}
$$

In fact, our reasoning after (4.5) define $\frac{\partial \mathcal{F}}{\partial T_{n}}$ up to integration "constants", which can depend only on $T$ 's, but not on a's. Actually, $\mathcal{F}$ is defined modulo addition of any quadratic function of $T$ 's, $\sum_{m, n=-\infty}^{\infty} f_{m n} T_{m} T_{n}$ with constant coefficients $f_{m n}$. In practice this is never important, except for one place: the integral for $\frac{\partial \mathcal{F}}{\partial T_{0}}$ in (4.6) is actually divergent, since $d \mathcal{S}(\xi)$ has poles at $\xi_{ \pm}$. However, there was no singularity of this kind in (4.5): for $n=0$ it was just $\oint_{\text {cut }} \log \frac{\xi-\xi_{+}}{\xi-\xi_{-}} d \omega_{i}(\xi)=2 \pi \int_{\xi_{-}}^{\xi_{+}} d \omega_{i}$. Accordingly, the divergent part of $\frac{\partial \mathcal{F}}{\partial T_{0}}$ can be absorbed in allowed redefinition of $\mathcal{F}$.

4 The easiest way to see the singularity is to note that the matrix $\sigma_{k i} \equiv \sum_{m=-\infty}^{\infty} T_{m} \sigma_{k i}^{(m)}$ is vanishing when all $T_{n}=0$, thus $\sigma_{i k}^{-1}$ in (3.12) does not exist. It is also clear that whenever $\# k=p=\# i$ in (3.12) there can be no extra constraints (like the homogeneity condition) for the functions $h_{k}\left(a_{i}\right)$ to satisfy: they describe a non-degenerate change of variables. It may be enough, however, to introduce just one extra variable (like $T_{0}, T_{1}$, or - differently $\left.h_{0}\right)$ to make (3.12) consistent with homogeneity constraint - and thus allow some simple truncation of $\mathcal{F}$ to exist. This is the trick, often used in considerations of Seiberg-Witten theory: see [8] as well as s.4.4 and s.6 below. 


\section{$4.2 \quad$ Homogeneity of $\mathcal{F}$}

Homogeneity property (3.15) implies that the prepotential $\mathcal{F}\left(a_{i}, T_{n}\right)$ always is a homogeneous function of degree 2 :

$$
\mathcal{F}=\frac{1}{2}\left(\sum_{i=1}^{p} a_{i} \frac{\partial \mathcal{F}}{\partial a_{i}}+\sum_{n=-\infty}^{\infty} T_{n} \frac{\partial \mathcal{F}}{\partial T_{n}}\right) .
$$

Indeed, assuming that (4.8) is true, and making use of (4.7) and (4.6),

$$
\begin{gathered}
\mathcal{F}=\frac{1}{2} \sum_{i=1}^{p} a_{i} \oint_{B_{i}} d \mathcal{S}+\frac{1}{2} \sum_{n>0} \frac{2 \pi i}{n} T_{ \pm n} \operatorname{res}_{\xi_{ \pm}}\left(\xi-\xi_{ \pm}\right)^{-n} d \mathcal{S}(\xi)+\pi T_{0} \int_{\xi_{-}}^{\xi_{+}} d \mathcal{S} \stackrel{(3.14)}{=} \\
=\frac{1}{2} \sum_{i, j=1}^{p} a_{i} a_{j} \mathcal{T}_{i j}(h)+\sum_{i=1}^{p} \sum_{n=-\infty}^{\infty} a_{i} T_{n} \kappa_{i}^{(n)}(h)+\frac{1}{2} \sum_{m, n} \frac{2 \pi i}{n} T_{ \pm n} T_{m} \operatorname{res}_{\xi_{ \pm}}\left[\left(\xi-\xi_{ \pm}\right)^{-n} d \Omega_{m}(\xi)\right] .
\end{gathered}
$$

All the coefficients at the r.h.s. (including the residue in the last term) are functions of moduli $h$ only, thus - given (3.15) - what we obtain is indeed homogeneous of degree 2 and our assumption (4.8) is a posteriori justified.

"Explicit formula" (4.9) should be of course supplemented by relations (3.12) and (3.7):

$$
\frac{\partial h_{k}}{\partial a_{j}}=\sigma_{j k}^{-1}(h), \quad \frac{\partial h_{k}}{\partial T_{n}}=\sum_{i=1}^{p} c_{i}^{(n)}(h) \sigma_{i k}^{-1}(h) .
$$

From (3.14) one can also obtain:

$$
a_{i}=\oint_{A_{i}} d \mathcal{S}, \quad T_{ \pm n}=\frac{1}{2 \pi i} \oint\left(\xi-\xi_{ \pm}\right)^{+n} d \mathcal{S}
$$

(in the second case the property (ii) of $d \Omega$ is used). Substituting (4.6), (4.7) and (4.11) back into (4.8), we get:

$$
\begin{gathered}
\mathcal{F}=\frac{1}{2} \sum_{i=1}^{p} \oint_{A_{i}} d \mathcal{S} \oint_{B_{i}} d \mathcal{S}+\sum_{n>0} \frac{1}{4 \pi i n} \oint_{\xi_{ \pm}}\left(\xi-\xi_{ \pm}\right)^{+n} d \mathcal{S} \oint_{\xi_{ \pm}} \frac{d \mathcal{S}}{\left(\xi-\xi_{ \pm}\right)^{n}}+\frac{1}{2} \oint_{\xi_{+}} d \mathcal{S} \int_{\xi_{-}}^{\xi_{+}} d \mathcal{S}= \\
=\frac{1}{2} \sum_{i=1}^{p} \oint_{A_{i}} d \mathcal{S} \oint_{B_{i}} d \mathcal{S}+\frac{1}{2 \pi i} \iint \log \left(\xi-\xi^{\prime}\right) d \mathcal{S}(\xi) d \mathcal{S}\left(\xi^{\prime}\right) .
\end{gathered}
$$

Note, that while $\frac{\partial \mathcal{F}}{\partial T_{n}}$ in (4.6) and $T_{n}$ in (4.11) depend on the choice of coordinate system (e.g. change under rescaling $\xi \rightarrow$ const $\cdot \xi)$, this dependence cancels in (4.12).

\subsection{Discussion}

Eq.(4.12) is a kind of analogue of Hirota bilinear equation for $\mathrm{KP} /$ Toda $\tau$-function, and in this analogy the 1-differential $d \mathcal{S}(\xi)$ substitutes the Baker-Akhiezer $\frac{1}{2}$-differential $\Psi(\xi)$. Note that $(4.12)$ 
depends on the data $\left\{\right.$ the curve $\mathcal{C}$, points $\xi_{ \pm}$, coordinate system $\}$- which is exactly the data, specifying particular solution to Hirota equations. This reflects the fact that the set of Whitham equations depends on both, original system (e.g. KP/Toda hierarchy) and its solution. The main drawback of (4.12) in its present form is the lack of alternative expression of $d \mathcal{S}(\xi)$ through $\mathcal{F}$ while for the usual $\mathrm{KP} /$ Toda Baker-Akhiezer function

$$
\Psi(\xi)=\frac{\tau\left(t_{ \pm n}-\frac{1}{n}\left(\xi-\xi_{ \pm}\right)^{n}\right)}{\tau\left(t_{ \pm n}\right)} \exp \left(\sum_{n} t_{ \pm n}\left(\xi-\xi_{ \pm}\right)^{-n}\right)
$$

This is what makes the theory of prepotential with infinitely many time-variables - at the present stage - dependent on the formalism of KP/Toda-hierarchies, involving spectral curves (not hypersurfaces), 1-differentials etc - and makes applications beyond such framework not straightforward. Such applications, of course, exist: in the context of Seiberg-Witten theory the simplest example is its reformulation in terms of Calabi-Yau manifolds [12, 13, 14].

Moreover, even in the case of spectral curves, above consideration is not exhaustive, because it relies heavily on 1-differentials, rather than on 2-differentials, which are better suited for consideration of generic moduli spaces of complex curves. In fact, the whole theory of prepotential is the one about cohomology groups, more exactly Hodge structures over the moduli spaces and is often formulated this way in considerations starting from topological field theories, see $[6,5]$ and references therein.

\subsection{Homogeneity property in terms of $d \mathcal{S}$}

Eq.(4.8) can be reexpressed in terms of $d \mathcal{S}$, what is useful both for applications and for generalizations. For this, differentiate (4.8) w.r.to $h_{k}$, keeping all the $T_{n}$ fixed:

$$
\frac{\partial}{\partial h_{k}}\left(2 \mathcal{F}-\sum_{i=1}^{p} a_{i} \frac{\partial F}{\partial a_{i}}\right)=\sum_{i=1}^{p}\left(\frac{\partial a_{i}}{\partial h_{k}} \frac{\partial \mathcal{F}}{\partial a_{i}}-a_{i} \frac{\partial}{\partial h_{k}} \frac{\partial \mathcal{F}}{\partial a_{i}}\right)=\sum_{n=-\infty}^{+\infty} T_{n} \frac{\partial}{\partial h_{k}} \frac{\partial \mathcal{F}}{\partial T_{n}}
$$

Using (4.3), the l.h.s. can be also rewritten as

$$
\sum_{i=1}^{p}\left(\frac{\partial a_{i}}{\partial h_{k}} a_{i}^{D}-a_{i} \frac{\partial a_{i}^{D}}{\partial h_{k}}\right)=\sum_{i=1}^{p}\left(\oint_{A_{i}} \frac{\partial d \mathcal{S}}{\partial h_{k}} \oint_{B_{i}} d \mathcal{S}-\oint_{B_{i}} \frac{\partial d \mathcal{S}}{\partial h_{k}} \oint_{A_{i}} d \mathcal{S}\right)=\oint_{\text {sing }} \mathcal{S} \frac{\partial d \mathcal{S}}{\partial h_{k}} .
$$

where the integral at the r.h.s. runs around the singularities of $d \mathcal{S}$ and $\partial d \mathcal{S} / \partial h_{k}$. The last identity in (4.15) is valid for any (1,0)-differential $d \mathcal{S}$ (it results from integration of identity $d \mathcal{S} \wedge \frac{\partial d \mathcal{S}}{\partial h_{k}}=0$ over entire Riemann surface $\mathcal{C}$, comp.with footnote 3 ). Assuming that $d \mathcal{S}$ is meromorphic with

poles only at $\xi_{ \pm}$and $\frac{\partial d \mathcal{S}}{\partial h_{k}}$ is holomorphic, one can rewrite the r.h.s. of (4.15) in a more explicit 
form:

$$
\begin{gathered}
\text { l.h.s.of }(4.14) \stackrel{(4.3)}{=} \oint_{\text {sing }} \mathcal{S} \frac{\partial d \mathcal{S}}{\partial h_{k}}= \\
=\frac{1}{i} \oint_{\xi_{+}} d \mathcal{S}(\xi) \int_{\xi_{-}}^{\xi_{+}} \frac{\partial d \mathcal{S}\left(\xi^{\prime}\right)}{\partial h_{k}}+\sum_{n=1}^{\infty} \frac{1}{2 \pi i n}\left(\oint_{\xi_{+}}\left(\xi-\xi_{+}\right)^{n} d \mathcal{S}(\xi) \oint_{\xi_{+}}\left(\xi^{\prime}-\xi_{+}\right)^{-n} \frac{\partial d \mathcal{S}\left(\xi^{\prime}\right)}{\partial h_{k}}+\right. \\
\left.+\oint_{\xi_{-}}\left(\xi-\xi_{-}\right)^{n} d \mathcal{S}(\xi) \oint_{\xi_{-}}\left(\xi^{\prime}-\xi_{-}\right)^{-n} \frac{\partial d \mathcal{S}\left(\xi^{\prime}\right)}{\partial h_{k}}\right) .
\end{gathered}
$$

It is easy to recognize in this expression the r.h.s. of (4.14) with (4.6) and (4.11) substituted in it.

Holomorphicity of $\frac{\partial d \mathcal{S}}{\partial h_{k}}$, required in this derivation, is exactly the requirement (2.1), which is in fact the crucial reason for (4.2) and (4.3) to hold. Thus, as we see once again, from certain point of view the $T$-dependence does not introduce anything essentially new: given $d S$ that satisfies $(2.2)$ and thus (4.3), eqs.(4.6) and (4.11) can be just used to define what are $T_{n}$ and $\frac{\partial \mathcal{F}}{\partial T_{n}}$.

Moreover, the holomorphicity requirement (2.1) implies that so defined $T_{ \pm n}=\oint_{\xi_{ \pm}}\left(\xi-\xi_{ \pm}\right)^{n} d S(\xi)$ are independent of $h_{k}$ (because $\operatorname{res}_{\xi_{ \pm}}\left(\xi-\xi_{ \pm}\right)^{n} d \omega_{j}(\xi)=0$ for any $\left.n \geq 0\right)$ - and thus (4.16) can be explicitly integrated over $h_{k}$ : it is enough to substitute $\frac{\partial d \mathcal{S}\left(\xi^{\prime}\right)}{\partial h_{k}} \rightarrow d \mathcal{S}\left(\xi^{\prime}\right)$. In other words, we obtain:

$$
\begin{gathered}
2 \mathcal{F}-\sum_{i=1}^{p} a_{i} \frac{\partial \mathcal{F}}{\partial a_{i}}= \\
=\frac{1}{i} \oint_{\xi_{+}} d \mathcal{S} \int_{\xi_{-}}^{\xi_{+}} d \mathcal{S}+\sum_{n=1}^{\infty} \frac{1}{2 \pi i n} \sum_{\alpha= \pm} \oint_{\xi_{\alpha}}\left(\xi-\xi_{\alpha}\right)^{n} d \mathcal{S}(\xi) \oint_{\xi_{\alpha}}\left(\xi^{\prime}-\xi_{\alpha}\right)^{-n} d \mathcal{S}\left(\xi^{\prime}\right) .
\end{gathered}
$$

Once a particular solution $d S\left(h_{k}\right)$ of (2.1) is given, the r.h.s. of (4.17) is explicit function of $h_{k}$.

Of course, concrete solution corresponds to concrete values of time-variables $T_{n}$ - and in this sense eq.(4.17) is somewhat artificial. However, according to [8], such approach, based on (4.12) and (4.8) is instead useful for comparison of the abstract prepotential theory with numerous particular results, obtained by more sophisticated methods $[15,9]$, see also s.6 below. Moreover it can be immediately generalized beyond spectral curves - to (certain) complex manifolds of arbitrary dimension.

\section{Prepotential beyond one complex dimension}

We can now reverse the logic and consider eq.(4.12) as the starting point instead of eq.(2.1). The disadvantage of such approach is that we can loose understanding of what is original model, to which our consideration is a Whitham approximation - in the case of (2.2) it was a system of $\mathrm{KP} /$ Toda family (and - less obviously - the $N=2$ gauge models in $4 d$ ). Of course, this is also the 
main advantage: this opens the way to considerations in the spirit of integrability theory beyond the $\mathrm{KP} /$ Toda framework. As we shall see, there is no problem of developing the literal analogue of above Whitham theory for certain complex manifolds of any dimension instead of complex curves (with immediate examples provided by K3 and Calabi-Yau manifolds). The analogue of entire $\mathrm{KP} /$ Toda theory for these examples should be intrinsically stringy and probably include multi-loop groups, but the Whitham approximation is very similar to $\mathrm{KP} /$ Toda case - as one expects a kind of simplified universal description emerges in the "slow" or "low-energy" limit. With no surprise this higher-dimensional extension of Whitham formalism is very close to "special geometry" [16], as well as to the abstract constructions of [5] and [6].

The content of this section is very much a repetition of what was said in s.4: but now the accents and the whole logic are different - and just this rephrasing makes possible a much wider applications of essentially the same formulas.

\subsection{Notations and definition}

Consider a family $\mathcal{M}(h)$ of complex manifolds $M$ of complex dimension $d$ (in the previous sections $d=1$ and $\mathcal{M}(h)$ are some families of spectral curves, $M=\mathcal{C}$ ). The family is parametrized by some moduli $h_{k}, k=1, \ldots, K=\operatorname{dim}_{C} \mathcal{M}$. Let us fix some canonical system of $d$-cycles on $M:\left\{A_{i}, B_{i}\right\}$, $i=1, \ldots, p=\frac{1}{2} \operatorname{dim} H^{d}(M)$ with the intersection matrix $A_{i} \# B_{j}=\delta_{i j}, A_{i} \# A_{j}=B_{i} \# B_{j}=0$. Finaly, pick up some holomorphic $(d, 0)$-form $\Omega$ on every $M .{ }^{5}$ Its periods,

$$
a_{i}(h) \equiv \oint_{A_{i}} \Omega, \quad a_{i}^{D}(h) \equiv \oint_{B_{i}} \Omega
$$

are functions of moduli.

Consider now a variation $\delta \Omega$ of $\Omega$ with the change of parameters (moduli). $\delta \Omega$ is also a $(d, 0)$ form, not necessarily holomorphic. Still, always $\Omega \wedge \delta \Omega=0$ (just because $\Omega$ is a maximal-rank form), and integraion of this relation over entire $M$ gives:

$$
0=\int_{M} \Omega \wedge \delta \Omega=\sum_{i}\left(\oint_{A_{i}} \Omega \oint_{B_{i}} \delta \Omega-\oint_{A_{i}} \delta \Omega \oint_{B_{i}} \Omega\right)+\text { contribution from singularities. }
$$

Imagine that the last item at the r.h.s. - the contribution from singularities of $\Omega$ and $\delta \Omega$ is absent. Then we obtain from (5.2):

$$
\sum_{i} a_{i} \delta a_{i}^{D}=\sum a_{i}^{D} \delta a_{i}
$$

\footnotetext{
${ }^{5}$ It is clearly a restriction on $M$ that such $\Omega$ exists, examples of suitable $M$ are provided by $K 3(d=2)$ and Calabi-Yau $(d=3)$ manifolds. In our discussion below we shall see that this restriction can sometime be weekend, by admitting $\Omega$ 's with simple singularities. Additional requirements for $\Omega$-dependence on moduli - in the spirit of (2.1) - will be specified later.
} 
This implies that the prepotential, defined as -

$$
\mathcal{F} \equiv \frac{1}{2} \sum_{i} a_{i} a_{i}^{D}=\frac{1}{2} \sum_{i} \oint_{A_{i}} \Omega \oint_{B_{i}} \Omega
$$

possesses the following property:

$$
\delta \mathcal{F}=\frac{1}{2} \sum_{i}\left(a_{i} \delta a_{i}^{D}+a_{i}^{D} \delta a_{i}\right)=\sum_{i} a_{i}^{D} \delta a_{i} .
$$

If the freedom of variations is big enough, e.g. if $\# K=\operatorname{dim}_{C} \mathcal{M}$ is the same as $\# p=\frac{1}{2} \operatorname{dim} H^{d}(M)$, we conclude from this that

$$
a_{i}^{D}=\frac{\partial \mathcal{F}}{\partial a_{i}}
$$

and

$$
\mathcal{F}=\frac{1}{2} \sum_{i} a_{i} a_{i}^{D}=\frac{1}{2} \sum_{i} a_{i} \frac{\partial \mathcal{F}}{\partial a_{i}} .
$$

In other words, we can consider $a_{i}$ as independent variables, and introduce the prepotential $\mathcal{F}(a)$ by the rule (5.4) - and it will always be a homogeneous function of degree 2 - as follows from (5.7).

The two requirements, built into this simple construction, are:

(i) the absence of singularity contributions at the r.h.s. of (5.2);

(ii) the matching between the quantities of moduli and $A$-cycles,

$$
K \equiv \operatorname{dim}_{C} \mathcal{M}=p \equiv \frac{1}{2} \operatorname{dim} H^{d}(M)
$$

\subsection{Comments on requirement (i)}

The problem with this restriction is that variation of holomorphic object w.r.to moduli usually makes it singular - by the very definition of moduli of complex structure. Thus, even if $\Omega$ is free of singularities one should expect them to appear in $\delta \Omega$. The only way out would be to get the newly emerging poles canceled by zeroes of $\Omega$ - but often the space of holomorphic $\Omega$ 's is too small to allow for adequate adjustement.

Fortunately, requirement (i) can be made less restrictive. One can allow to consider $\Omega$ which is not holomorphic, but possesses simple singularities at isolated divisors. As a pay for this it is enough to enlarge the set of $A$-cycles, by adding the ones, wrapping around the singularity divisors, and also add all independent $B$-chains, connecting these divisors (such that $\left.\partial B=d i v_{1}-d i v_{2}\right) .{ }^{6}$ At

\footnotetext{
${ }^{6}$ For example, if $M$ is a complex curve $(d=1), \Omega$ can be a meromorphic (1,0)-differential with simple (order one) poles at some punctures $\xi_{\alpha}, \alpha=0,1, \ldots, r$. Then one should add $r$ circles around the points $\xi_{1}, \ldots, \xi_{r}$ to the set of $A$-cycles, and $r$ lines (cuts) connecting $\xi_{0}$ with $\xi_{1}, \ldots, \xi_{r}$ to the set of $B$-contours in eq.(5.2). Then the last term at the r.h.s. can be omitted in exchange for enlarging the sum in the first term.
} 
the same time residues at the simple singularities should be added to the set of moduli $\{h\}$, thus preserving the status of the second requirement (ii).

This prescription is still not complete, because the integrals over newly-added $B$-chains are divergent (because these end at the singularities of $\Omega$ ). However, the structure of divergencies is very simple: if a cut-off is introduced, the cut-off-dependent piece in $\mathcal{F}$ is exactly quadratic in the new moduli - and does not depend on the old ones. If one agrees to define the prepotential - which is generic homogeneous function of order two - modulo quadratic functions of moduli, the problem is resolved.

Thus the real meaning of constraint (i) is that $\delta \Omega$ should not introduce new singularities as compared to $\Omega$ - so that we do not need to introduce new cycles, thus new moduli, derivatives over which would provide new singularities etc etc. Since now the freedom to choose $\Omega$ is big enough, such special adjustement is usually available.

The non-simple singularities (higher-order poles at divisors) should be resolved - i.e. considered as a limit of several simple ones when the corresponding divisors tend to coincide. The corresponding $B$-chains shrink to zero in the limit, but integrals of $\Omega$ over them do not vanish, if $\Omega$ is indeed singular enough. This procedure of course depends on particular way to resolve the non-simple singularity. Essentially, if we want to allow the one of arbitrary type on the given divisor, it is necessary to introduce coordinate system in the vicinity of divisor and consider all the negative terms of Laurent expansion of $\Omega$ as moduli, and "weighted" integrals around the divisor as $A$-cycles. In the case of $d=1$, when the divisors are just points (punctures) one can easily recognize in this picture the definition of $\mathrm{KP} /$ Toda-induced Whitham prepotential in the form of (4.12): with one-parameter set of "time"-variables (Laurent expansion coefficients) for every puncture as additional moduli. As often happens, it is most natural from the point of view of string theory (integrability theory in this case) to put all the moduli in a single point (or two), but from the point of view of algebraic geometry it is better to redistribute them as simple singularities at infinitely many divisors.

Finally, singularities of $\Omega$ on subspaces of codimension higher than one do not contribute to eq.(5.2) at all - and often variation w.r.to moduli produces only singularities of such type as $d>1$.

\subsection{Requirement (ii)}

Thus, what essentially remains is the other requirement (ii) - the matching condition between the number of moduli and $A$-cycles. Since the procedures involved in resolution of (i) do not change this matching (they always add as many new moduli as new $A$-cycles), this requirement can be 
analyzed at the very beginning - before even introducing $\Omega$.

The Seiberg-Witten theory of $4 d$ low-energy $N=2$ SUSY gauge theories provides a lot of examples when such matching takes place. Most of them have immediate counterparts in the $1 d$ integrability theory $[3,17,18,19,20,4]$.

\section{Examples}

\subsection{Definitions}

In examples below we first list the curves $\mathcal{C}$, which are associated with particular $N=2$ SUSY gauge theories and - at the same time - with particular integrable systems of particles (see [4] for details and references). The "full" spectral curves are naturally defined by algebraic equations

$$
\mathcal{C}: \quad \operatorname{det}(t-L(z))=0
$$

with parameter $z$ being a coordinate on elliptic "bare spectral curve" $E(\tau)$ - which in many cases degenerates into a sphere (in a special double scaling limit as $\tau \rightarrow i \infty$ ). For every example we consider the "minimal" $d \mathcal{S}$ - a solution to $(2.1)$, defined by the formula ${ }^{7}$

$$
d \mathcal{S}_{\min } \cong 2 t d \omega_{0}(z) \stackrel{\tau \rightarrow i \infty}{\longrightarrow} \frac{t}{i \pi} d \log z
$$

where $d \omega_{0}(z)$ is distinguished canonical holomorphic 1-differential on $E(\tau)$, which in the scaling limit turns into $\frac{d z}{2 \pi i z}$ - or rather into $\frac{1}{2 \pi i}\left(\frac{d \xi}{\xi-\xi_{+}}-\frac{d \xi}{\xi-\xi_{-}}\right)$. This $d \mathcal{S}_{\min }$ is actually our $d \mathcal{S}\left(a_{i}, T_{n}\right)$ at the hypersurface where all the $T$-variables are vanishing - except for one or two, which are usually $T_{ \pm 1}$ or $T_{0}$. Given $d \mathcal{S}_{\min }$ and accepting the upside-down logic of section 5, one can use (4.12) to define the reduced prepotential

$$
\left.\mathcal{F}_{\text {red }}\left(a_{i}\right) \equiv \mathcal{F}\left(a_{i}, T_{n}\right)\right|_{\begin{array}{l}
T_{0} \text { or } T_{ \pm 1}=\text { const } \\
\text { all other } T_{n}=0
\end{array}},
$$

which is not homogeneous as a function of $a_{i}$, but instead satisfies

$$
\begin{gathered}
2 \mathcal{F}_{\text {red }}-\sum_{i=1}^{p} a_{i} \frac{\partial \mathcal{F}_{\text {red }}}{\partial a_{i}}=T_{0} \frac{\partial \mathcal{F}_{\text {red }}}{\partial T_{0}}+T_{1} \frac{\partial \mathcal{F}_{\text {red }}}{\partial T_{1}}+T_{-1} \frac{\partial \mathcal{F}_{\text {red }}}{\partial T_{-1}} \stackrel{(4.17)}{=} \\
=2 \pi \cdot \operatorname{res}_{\xi_{+}}\left[d \mathcal{S}_{\text {min }}\right] \int_{\xi_{-}}^{\xi_{+}} d \mathcal{S}_{\text {min }}+\sum_{\alpha= \pm} \operatorname{res}_{\xi_{\alpha}}\left[\left(\xi-\xi_{\alpha}\right) d \mathcal{S}_{\min }(\xi)\right] \oint_{\xi_{\alpha}} \frac{d \mathcal{S}_{\text {min }}(\xi)}{\xi-\xi_{\alpha}} .
\end{gathered}
$$

This was also the logic accepted in [8].

\footnotetext{
7 There are various (essentially equivalent) ways to characterize $d \mathcal{S}_{\min }$ : as a minimal solution to the constraint (2.1), as reduced $d \mathcal{S}(3.14)$, as $d^{-1}$ of symplectic form $d t \wedge d \omega_{0}$ on the Hitchin variety etc.
} 
Actually, we shall mostly concentrate on the case of $S L(2)$ group. Of most interest are relations in the chain pure gauge $N=2$ - pure gauge $N=4$ - Calabi-Yau compactification, which can be revealed already at the $S L(2)$ level. As the last example, we discuss the Calabi-Yau manifold, associated with $W P_{1,1,2,2,6}^{12}$. Related string-induced model is known [13] to reproduce $S U(2) N=2$ Seiberg-Witten/sine-Gordon theory in the conifold limit. Beyond conifold limit one rather expects it to be related in the same sense to the $S U(2) N=4 \mathrm{SW} /$ Calogero model: ${ }^{8}$ the prepotentials for the two - when written in appropriate variables - should be closely related, if not the same. Exact result should be looked for at the level of Picard-Fuchs equations. We demonstrate that certain similarity indeed exists, but exact equality hardly takes place.

\subsection{Comments}

As we already mentioned in the previous sections, (4.12) is essentially the same as (6.3):

$$
\begin{gathered}
\mathcal{F}_{\text {red }}\left(a_{i}\right)=\frac{1}{2} \sum_{i=1}^{p} a_{i} a_{i}^{D}+\pi T_{0} \int_{\xi_{-}}^{\xi_{+}} d \mathcal{S}_{\min }+\frac{1}{2} \sum_{\alpha} T_{\alpha 1} \oint_{\xi_{\alpha}} \frac{d \mathcal{S}_{\min }(\xi)}{\xi-\xi_{\alpha}}, \\
a_{i}=\oint_{A_{i}} d \mathcal{S}_{\min }, \quad T_{0}=\operatorname{res}_{\xi_{+}}\left[d \mathcal{S}_{\min }\right], \quad T_{1}=\operatorname{res}_{\xi_{\alpha}}\left[\left(\xi-\xi_{\alpha}\right) d \mathcal{S}_{\min }(\xi)\right] .
\end{gathered}
$$

This should be considered as a somewhat implicit formula for $\mathcal{F}_{\text {red }}\left(a_{i}\right)$ : eq.(6.4) define it as a function of $h_{k}$ (on which $d \mathcal{S}_{\text {min }}$ depends), rather than that of $a_{i}$ : eq.(6.5) should be resolved to give $h_{k}\left(a_{i}\right)$ and then the result is to be substituted into (6.4). It now follows from the general theory above that

$$
\oint_{B_{i}} d \mathcal{S}_{\min } \equiv a_{i}^{D}=\left.\frac{\partial \mathcal{F}}{\partial a_{i}}\right|_{T_{0}, T \alpha 1=\text { const }} .
$$

In fact, only $a_{i}, a_{i}^{D}$ and their dependence on $h_{k}$ have physical meaning - both in $4 d$ and $1 d$ models. ${ }^{9}$ From this point of view the shapes of the curves $\mathcal{C}\left(h_{k}\right)$ are not uniquely defined for the given model. As is well known since [2], different curves can produce the same periods $a_{i}(h), a_{i}^{D}(h)$ (with the same $h$-dependence). Obviously, a covering of original curve will reproduce the same periods - but it will be another curve. In eq.(6.1) this freedom is reflected by that of the choice of representation of the Lax operator for the given group.

Dependence of $a_{i}$ and $a_{i}^{D}$ on $h_{k}$ is usefully encoded in terms of the so-called Picard-Fuchs equations (PFE) - which immediately arise once integral formulas like $a_{i}\left(h_{k}\right)=\oint_{A_{i}} d \mathcal{S}_{\min }\left(h_{k}\right)$,

\footnotetext{
8 A related claim is made in a recent paper [21].

${ }^{9}$ It deserves reminding that moduli $h_{k}$ are vacuum expectation values of certain scalar fields and eigenvalues of Hamiltonians in $4 d$ and $1 d$ respectively. The periods $a_{i}$ and $a_{i}^{D}$ in $4 d$ are entering the mass mormula for the BPSsaturated states, which are physical (this is significant distinction of $N=2$ SUSY theories - normally the background fields in the Wilsonian action, like $a_{i}$ and $a_{i}^{D}$, are not physical observables). In $1 d$ the $a_{i}$ and $a_{i}^{D}$ are action integrals.
} 
$a_{i}^{D}\left(h_{k}\right)=\oint_{B_{i}} d \mathcal{S}_{\min }\left(h_{k}\right)$, are written down. At least naively, these equations are additional pieces of information as compared to the prepotential: the latter one is a function of $a_{i}$ and does not contain any reference to $h_{k}$. This is also reflected in the fact that details of differential geometry on particular curves $\mathcal{C}$ (and manifolds $M$, in general) are involved in the appearence of PFE (to say nothing about their derivation): occurence of the $\sigma_{k j}$ matrix in (3.12) is an (oversimplified) example. Of course, once all the PFE for some two models coincide, the same is true about their prepotentials - provided they exist: the PFE can be written down for any cohomology class - not just to the one represented by $d \mathcal{S}_{\min }\left(h_{k}\right)$. But if the PFE are written for this particular class - they can tell us a lot about the prepotential, which does exist because $d \mathcal{S}_{\min }$ satisfies (2.1). Similarly, in the framework of special geometry in higher dimensions - where again the prepotential exists by definition, - the PFE for cohomology class of $\Omega\left(h_{k}\right)$ can be used for its investigation.

\section{Examples of UV-infinite $4 d$ models: Riemann sphere as the bare spectral surface}

As explained in $[2,18,4]$, the $d=4$ models which are asymptotically free in the UV and acquire mass scale as result of dimensional transmutation, in the language of $1 d$ integrable systems correspond to the models with singular bare spectral surface. In the simplest setting this can be just a Riemann sphere with punctures: two for pure gauge $N=2$ SUSY models and more when extra matter multiplets are introduced. The "soft" UV regularization of the theory (resulting from its embedding into some UV-finite model) corresponds to blowing up the singularities (punctures), making the bare spectral surface into elliptic curve (torus) $E(\tau)$ : this will be the subject of our consideration in the $\mathrm{s} .8$ below.

\subsection{Original Seiberg-Witten (sine-Gordon) example. Curve and $d \mathcal{S}_{\min }$}

This basic example was introduced in [1] for description of (the low-energy limit of) pure $S U(2)$ gauge $N=2$ SUSY theory and considered from the point of view of integrability theory in [3]: the relevant universality class appears to be that of $1 d$ sine-Gordon model (a particle in cosine potential). The full spectral curve $\mathcal{C}$ is of genus $p=1$ and is defined by elliptic equation

$$
\tilde{y}^{2}=(\tilde{x}-u)\left(\tilde{x}^{2}-\Lambda^{4}\right), \quad u=2 h_{2} \quad\left(=2 h_{2}^{N=2}=2 h_{2}^{\mathrm{SG}}\right) .
$$


The degenerated bare spectral curve is a 1-punctured sphere, and the associated $d \mathcal{S}_{\min }$ is (see s.8 below for details of degeneration scaling limit):

$$
d \mathcal{S}_{\min } \cong \frac{1}{\pi \sqrt{2}} \frac{\tilde{x}-u}{\tilde{y}(\tilde{x})} d \tilde{x}=\frac{\Lambda}{\pi \sqrt{2}} \sqrt{\frac{u}{\Lambda^{2}}-\cos \varphi} d \varphi=\frac{1}{2 \pi i} \sqrt{2 u+\Lambda^{2}\left(z+\frac{1}{z}\right)} \frac{d z}{z} .
$$

Obviously,

$$
\frac{\partial d \mathcal{S}_{\min }}{\partial u}=-\frac{1}{2 \pi \sqrt{2}} \frac{d x}{\tilde{y}(x)}=-\frac{1}{2 \pi \sqrt{2}} d v(x)
$$

- in accordance with (2.2). This $d \mathcal{S}_{\min }$ has double pole at the branch point $x=\infty$, with vanishing residue. Let us assume that coordinate in the vicinity of $x=\infty$ is $\xi-\xi_{\infty}=\sqrt{\Lambda^{2} / x}$. Then $d \mathcal{S}_{\min }=\frac{\sqrt{2} \Lambda}{\pi} d \hat{\Omega}_{1}$, so that $T_{1}=\frac{\sqrt{2}}{\pi} \Lambda$, and all other $T_{n}=0$.

\subsection{Prepotential}

According to (6.4)

$$
\mathcal{F}_{\text {red }}(a)=\frac{1}{2} a a_{D}-\frac{i u}{\pi}
$$

Here:

$$
\begin{aligned}
a=\oint_{A} d \mathcal{S}_{\min } & =\frac{\sqrt{2}}{\pi} \int_{-\Lambda^{2}}^{+\Lambda^{2}} \frac{x-u}{\tilde{y}(x)} d x=\frac{\sqrt{2}}{\pi} \int_{-\Lambda^{2}}^{+\Lambda^{2}} \sqrt{\frac{x-u}{x^{2}-\Lambda^{4}}} d x, \\
a^{D} & =\oint_{B} d \mathcal{S}_{\min }=\frac{\sqrt{2}}{\pi} \int_{\Lambda^{2}}^{u} \sqrt{\frac{x-u}{x^{2}-\Lambda^{4}}} d x
\end{aligned}
$$

and $-\frac{i}{\pi}=\frac{1}{2} \cdot\left(\frac{\sqrt{2}}{\pi} \Lambda\right)^{2} \cdot 2 \pi i \cdot\left(-\frac{u}{2 \Lambda^{2}}\right)$. By definition of $\mathcal{F}_{\text {red }}$,

$$
a^{D}=\frac{\partial \mathcal{F}_{\mathrm{red}}}{\partial a}
$$

In the large- $a$ (large- $u$ ) limit things simplify a lot:

$$
a^{D} \sim \frac{2 i}{\pi} a \log a, \quad u \sim \frac{1}{2} a^{2},
$$

and (7.3) is just the function

$$
\mathcal{F}_{\text {red }}(a)=\int^{a} a^{D} d a \sim \frac{2 i}{\pi} \int^{a} a \log a d a=\frac{i}{\pi} a^{2} \log a-\frac{i}{2 \pi} a^{2}=\frac{1}{2} a a_{D}-\frac{i u}{\pi} .
$$

For generic $a$ these formulas are more involved: to check (7.5) given (7.3) one should check that the integrals (7.4) satisfy

$$
a^{D}=\frac{\partial \mathcal{F}_{\mathrm{red}}}{\partial a}=\frac{1}{2} a^{D}+\frac{1}{2} a \frac{\partial a_{D}}{\partial a}+\frac{1}{i \pi} \frac{\partial u}{\partial a}
$$

Since

$$
\frac{\partial a}{\partial u}=-\frac{1}{\pi \sqrt{2}} \int_{-\Lambda^{2}}^{\Lambda^{2}} \frac{d x}{\tilde{y}(x)}=-\frac{1}{\pi \sqrt{2}} \int_{-\Lambda^{2}}^{\Lambda^{2}} \frac{d x}{\sqrt{(x-u)\left(x^{2}-\Lambda^{4}\right)}}
$$


eq.(7.8) is an identity for elliptic integrals. Of course it is immediate corollary of our usual argument

$$
\begin{aligned}
& \left(d v(x)=\frac{d x}{\tilde{y}(x)}\right): \\
& \quad 0=\int_{\mathcal{C}} d \mathcal{S}_{\min } \wedge d v=\oint_{A} d \mathcal{S}_{\min } \oint_{B} d v-\oint_{B} d \mathcal{S}_{\min } \oint_{A} d v-\operatorname{res}_{\infty}\left[\mathcal{S}_{\min }(\tilde{x}) d v(\tilde{x})\right] .
\end{aligned}
$$

The homogeneity equation (6.3) for (7.3) is just immediate:

$$
2 \mathcal{F}_{\text {red }}-a \frac{\partial \mathcal{F}_{\text {red }}}{\partial a}=\left(a a^{D}-\frac{2 i u}{\pi}\right)-a a^{D}=-\frac{2 i u}{\pi} .
$$

\subsection{Picard-Fuchs equation}

In the case of $S L(2)$, when there is a single module $h_{2}$, eq.(4.17) - i.e. eq.(7.10) - possesses one more useful interpretation [15]: in terms of Picard-Fuchs equations.

The starting point is relation of the form

$$
\mathcal{D}\left(h_{k}, \frac{\partial}{\partial h_{k}}\right) \cong 0
$$

for some differential operator $\mathcal{D}(h, \partial / \partial h)$, peculiar for the given moduli space (i.e. the family of spectral curves under consideration). In the particular case of (7.2) this identity is

$$
\left(\frac{\partial^{2}}{\partial u^{2}}-\frac{1}{4\left(\Lambda^{4}-u^{2}\right)}\right) d \mathcal{S}_{\min } \cong 0
$$

Indeed, the l.h.s. is proportional to the total derivative:

$$
0 \cong 2 d \sqrt{\frac{\tilde{x}^{2}-\Lambda^{4}}{\tilde{x}-u}}=\frac{\tilde{x}^{2}-2 u \tilde{x}+\Lambda^{4}}{\tilde{x}-u} \frac{d \tilde{x}}{\tilde{y}(\tilde{x})} \sim d \mathcal{S}_{\min }-4\left(\Lambda^{4}-u^{2}\right) \frac{\partial^{2}}{\partial u^{2}} d \mathcal{S}_{\min }
$$

As a relation in cohomologies, (7.11) is essentially an equation for the periods of $d \mathcal{S}_{\text {min }}$ :

$$
\mathcal{D}\left(\frac{\partial}{\partial h_{k}}\right) a_{i}=\mathcal{D}\left(\frac{\partial}{\partial h_{k}}\right) a_{i}^{D}=0 .
$$

When there is only one $h_{k}$ and $\mathcal{D}$ is a second order operator - as is the case for $(7.12)^{10}$ -

$$
\mathcal{D}\left(\frac{\partial}{\partial h}\right)=\frac{\partial^{2}}{\partial h^{2}}+U(h) \frac{\partial}{\partial h}+V(h),
$$

this can be transformed into a closed equation for the Wronskian

$$
W \equiv a \frac{\partial a^{D}}{\partial h}-\frac{\partial a}{\partial h} a^{D}=\frac{\partial}{\partial h}\left(2 \mathcal{F}-a \frac{\partial \mathcal{F}}{\partial a}\right):
$$

10 The reason for this is simple: in such case all the three differentials $d S, \frac{\partial}{\partial h} d S$ and $\frac{\partial^{2}}{\partial h^{2}} d S$ have vanishing residues, and in addition the spectral surface is of genus one,- therefore their cohomology classes are distinguished by only two periods, thus a linear relation should exist between the three classes. As soon as non-trivial residues are allowed at $r+1$ points and genus is $p$, the naive Picard-Fuchs equations should include $r+2 p$ derivatives. 


$$
\frac{\partial W}{\partial h}=a \frac{\partial^{2} a^{D}}{\partial h^{2}}-\frac{\partial^{2} a}{\partial h^{2}} a^{D}=-U(h) W,
$$

i.s. $W(h)=$ const $\cdot \exp -\int^{h} U\left(h^{\prime}\right) d h^{\prime}$ and

$$
2 \mathcal{F}-a \frac{\partial F}{\partial a}=\text { const } \cdot \int^{h} d h^{\prime \prime} \exp \left(-\int^{h^{\prime \prime}} U\left(h^{\prime}\right) d h^{\prime}\right)+\text { const' }^{\prime}
$$

In the particular case of (7.12) $U(h)=0$, and the r.h.s. of (7.15) is just const $\cdot h+$ const $^{\prime}$. The constants are not fixed by Picard-Fuchs equations, since they depend on normalizations of $d \mathcal{S}$ and the quasiclassical $\tau$-function $e^{\mathcal{F}}$.

From (7.12) one can immediately obtain the asymptotics of periods $\oint d \mathcal{S}_{\min }$. For large $u$ one can neglect $\Lambda^{4}$ and get $\oint d \mathcal{S}_{\min } \sim u^{\kappa}$, where $\kappa(\kappa-1)=-\frac{1}{4}$. This equation has double root $\kappa=\frac{1}{2}$, therefore allowed asymptotics are $\sqrt{u}$ and $\sqrt{u} \log u$ - in accordance with (7.6). For small $u$,

$$
\oint d \mathcal{S}_{\min }=\sum_{n=0}^{\infty} \gamma_{n}\left(\frac{u}{\Lambda^{2}}\right)^{n}
$$

and (7.12) provides the recurrent relation:

$$
\gamma_{n}=\frac{\left(n-\frac{1}{2}\right)^{2}}{n(n-1)} \gamma_{n-2}, \quad \text { i.e. } \quad \gamma_{2 n}=\frac{4^{n}}{(2 n) !}\left(\frac{\Gamma\left(n+\frac{1}{4}\right)}{\Gamma\left(\frac{1}{4}\right)}\right)^{2} \gamma_{0}, \quad \gamma_{2 n+1}=\frac{4^{n}}{(2 n+1) !}\left(\frac{\Gamma\left(n+\frac{3}{4}\right)}{\Gamma\left(\frac{3}{4}\right)}\right)^{2} \gamma_{1}
$$

Though eqs. of the type (7.11) usually exist on moduli spaces of compact complex surfaces, their explicit form - as well as the possibility to lift them to some identity for the prepotential $\mathcal{F}$ - is obscure in generic setting. Therefore, generalization of above reasoning for higher-rank groups - though possible - is rather involved, in any case it is far more sophisticated than the approach based on the theory of prepotential. Picard-Fuchs equations remain, however, the main available tool when the spectral surfaces are of non-unit complex dimension (like in the case with Calabi-Yau manifolds) - and alternatives are still underdeveloped.

\subsection{Toda-chain for $S L\left(N_{c}\right)$}

We now proceed to example of pure gauge $N=2$ SUSY theory with the gauge group $S U\left(N_{c}\right)$. According to $[22,23,24,17,4]$ the curve $\mathcal{C}$ in this case is of genus $p=\operatorname{rank}\left(S L\left(N_{c}\right)\right)=N_{c}-1$, and is given by the equation:

$$
z+\frac{1}{z}=2 P_{N_{c}}(t) \equiv \Lambda^{-N_{c}} \sum_{l=0}^{N_{c}} s_{l}(h) t^{N_{c}-l} .
$$

Here $s_{l}(h)$ are Schur polinomials, see [4] for details. It is important that $s_{1}(h)=h_{1}=0$ for $S L\left(N_{c}\right)$ (but not for $G L\left(N_{c}\right)$ ). Introducing $Y=\frac{1}{2}\left(z-\frac{1}{z}\right)$, one can rewrite this in hyperelliptic form:

$$
Y^{2}=P_{N_{c}}^{2}(t)-1
$$


Holomorphic 1-differentials on the hyperelliptic curve are [25] (linear combinations of) $d v_{j}=\frac{t^{j-1}}{Y(t)} d t$, $i \leq j \leq N_{c}-1=p$. (The basis $\left\{d v_{j}\right\}$ is not canonical, $\oint_{A_{i}} d v_{j} \neq \delta_{i j}$ ). According to (6.2),

$$
d \mathcal{S}_{\min } \cong \frac{t}{i \pi} d \log z=\frac{t}{i \pi} d \log \left(Y(t)+P_{N_{c}}(t)\right) .
$$

This indeed satisfies (2.2):

$$
\begin{gathered}
i \pi \frac{\partial d \mathcal{S}_{\min }}{\partial h_{k}} \cong t d \frac{\partial P_{N_{c}}(t) / \partial h_{k}}{Y(t)} \cong-\frac{\partial P_{N_{c}}(t)}{\partial h_{k}} \frac{d t}{Y(t)}= \\
=-\frac{1}{2} \Lambda^{-N_{c}} \sum_{l=2}^{N_{c}} \frac{\partial s_{l}(h)}{\partial h_{k}} \frac{t^{N_{c}-l} d t}{Y(t)}=-\frac{1}{2} \Lambda^{-N_{c}} \sum_{j=1}^{N_{c}-1} \frac{\partial s_{N_{c}+1-j}}{\partial h_{k}} d v_{j}(t) .
\end{gathered}
$$

The items with $l=0,1$ (i.e. with $j=N_{c}, N_{c}+1$ ) are absent at the r.h.s., since $s_{0}(h)=1$ and $s_{1}(h)=h_{1}=0 .{ }^{11}$

$d \mathcal{S}_{\min }$ has singularities at $z=0$ and $z=\infty$, in both cases $t=\infty$, which is not a branching point. The proper coordinate in the vicinity of singularities is $\xi=t^{-1}$, and

$$
d \mathcal{S}_{\mathrm{red}} \cong \frac{t}{i \pi} \frac{d z}{z}=\frac{t}{i \pi}\left(d \log t^{N_{c}}+O\left(t^{-2}\right)\right)=\frac{N_{c}}{i \pi} d t\left(1+O\left(t^{-2}\right)=\frac{i N_{c}}{\pi} \frac{d \xi}{\xi^{2}}\left(1+O\left(\xi^{2}\right)\right) .\right.
$$

From this we conclude that $d \mathcal{S}_{\min }$ corresponds to the choice $T_{1} \sim N_{c}$ and all other $T_{n}=0$.

In the particular case of $S L(2)$, the curve $\mathcal{C}_{S L(2)}$ considered in this section, does not coincide with that from s.7.1 (just the double ratios are different). Instead [2] one is the double cover of another, with the standard KP $\leftrightarrow$ Toda transformation $\tilde{x}=-\frac{\Lambda^{2}}{2}\left(z+\frac{1}{z}\right)$. The periods $a(h)$ and $a^{D}(h)$ are of course the same.

When $N_{f}$ matter $N=2$ supermultiplets in the fundamental representation of the gauge group are introduced, the equations (7.16) and (7.18) change for [24]:

$$
\begin{aligned}
& z+\frac{1}{z}=\frac{2 P_{N_{c}}(t)}{\sqrt{Q_{N_{f}}(t)}} \\
& d \mathcal{S}_{\min } \cong \frac{t}{i \pi} d \log z .
\end{aligned}
$$

with the same $P_{N_{c}}(t)$ and a new polinomial $Q_{N_{f}}(t)$ of degree $N_{f}$, which depends on the masses of matter multiplets. This can be also rewritten as

$$
\hat{z}+\frac{Q_{N_{f}}(t)}{\hat{z}}=2 P_{N_{c}}(t),
$$

11 Using the definition of Schur polinomials, $\sum_{l} s_{l}(h) t^{-l}=\exp \left(-\sum_{k} h_{k} t^{-k}\right)$, and its immediate corollary, $\frac{\partial s_{l}(h)}{\partial h_{k}}=-s_{l-k}(h)$, the chain of identites (7.19) can be further continued to give

$$
i \pi \frac{\partial d \mathcal{S}_{\min }}{\partial h_{k}} \cong+\frac{1}{2} \Lambda^{-N_{c}} \sum_{l} s_{l-k}(h) \frac{t^{N_{c}-l} d t}{Y(t)}=\left[t^{-k} P_{N_{c}}(t)\right]_{+} \frac{d t}{Y(t)},
$$

where the projector is defined by $\left[\sum_{n=-\infty}^{+\infty} u_{n} t^{n}\right]_{+} \equiv \sum_{n=0}^{+\infty} u_{n} t^{n}$. 
but then

$$
d \mathcal{S}_{\min }=\frac{t}{i \pi} d \log \left(\frac{\hat{z}}{\sqrt{Q_{N_{f}}(t)}}\right) .
$$

$d \mathcal{S}_{\min }$ now has extra simple poles, with residues proportional to the masses [2]. In such case the integral (6.4) for the prepotential diverges logarithmically, but the coefficients of divergent part are pure quadratic in masses, thus integrals can be easily regularized in controllable way. The periods are finite.

\section{UV-finite $4 d$ models and the $1 d$ Calogero system: Elliptic curve as the bare spectral surface}

The next set of examples is provided by gauge $N=2$ SUSY theories with additional matter multiplet in the adjoint representation of the gauge group $[2,18]$. As the mass $m$ of the mupltiplet changes from $m=\infty$ to $m=0$ the supersymmetry increases from $N=2$ to $N=4$. In the framework of integrability theory this flow is described by Calogero system [19, 20,4] with the role of $m$ played by the coupling constant $g \sim m$. The curve $\mathcal{C}$ is represented as a covering over elliptic "bare spectral curve" $E(\tau)$. This latter one can be parametrized either with a "flat" coordinate $\xi$ (so that the relevant objects are double periodic functions of $\xi$ ), or - alternatively - by elliptic equation

$$
E(\tau): \quad y^{2}=\prod_{a=1}^{3}\left(x-\hat{e}_{a}(\tau)\right)
$$

and

$$
d \omega_{0}=\frac{d \xi}{w_{1}}=\frac{1}{2 \pi} \frac{d x}{y(x)}
$$

Generic theory is rather sophisticated $[18,4]$, and in this paper we concentrate on the simplest case of the gauge group $S U(2)$.

\subsection{The case of the $S L(2)$ group. Curve, $d \mathcal{S}_{\min }$ and the prepotential}

In this case (6.1) says just:

$$
t^{2}-h_{2}=g^{2} \wp(\xi)=-\frac{m^{2}}{8} x
$$

thus $\mathcal{C}$ is a 2 -sheet covering of $E(\tau)$, and

$$
d \mathcal{S}_{\min } \cong 2 t d \omega_{0}=\frac{2}{w_{1}} \sqrt{h_{2}+g^{2} \wp(\xi)} d \xi=\frac{1}{\pi} \frac{\sqrt{h_{2}-\frac{m^{2}}{8} x}}{y(x)} d x .
$$


Here

$$
\left(\frac{\pi}{w_{1}}\right)^{2} x \equiv \wp(\xi) \equiv \frac{1}{\xi^{2}}+\sum_{\substack{-\infty<N, M<\infty \\ N^{2}+M^{2} \neq 0}}\left(\frac{1}{\left(\xi+N w_{1}+M w_{2}\right)^{2}}-\frac{1}{\left(N w_{1}+M w_{2}\right)^{2}}\right),
$$

is the doubly periodic Weierstrass $\wp$-function on the "bare spectral curve" $E(\tau), \quad \tau=\frac{w_{2}}{w_{1}}$, which satisfies the equation

$$
\frac{1}{4}\left[\wp^{\prime}(\xi)\right]^{2}=\prod_{a=1}^{3}\left(\wp(\xi)-e_{a}\right), \quad \hat{e}_{a}(\tau) \equiv\left(\frac{w_{1}}{\pi}\right)^{2} e_{a}\left(w_{1}, w_{2}\right), \quad \sum_{a=1}^{3} \hat{e}_{a}(\tau)=0
$$

Note that our $w_{1,2}$ are periods, not half-periods of $\wp(\xi)$. The coupling constant $g$ is proportional to the mass $m$ :

$$
g^{2}=\frac{m^{2}}{8}\left(\frac{i w_{1}}{\pi}\right)^{2}
$$

See [4] for more details.

The entire curve $\mathcal{C}$ is a double covering of $E(\tau)$, but periods of $d \mathcal{S}_{\min }$ - as is obvious from (8.4) - can be evaluated as if it was defined on an auxiliary genus-one curve

$$
\hat{\mathcal{C}}: \quad \hat{y}^{2}=\left(h_{2}-\frac{m^{2}}{8} x\right) \prod_{a=1}^{3}\left(x-\hat{e}_{a}(\tau)\right) .
$$

Obviously,

$$
d \mathcal{S}_{\min }=\frac{1}{\pi} \frac{h_{2}-\frac{m^{2}}{8} x}{\hat{y}(x)} d x
$$

and $\frac{\partial d \mathcal{S}_{\min }}{\partial h_{2}}=\frac{1}{2 \pi} \frac{d x}{\hat{y}(x)}$ is a holomorphic 1-differential on $\hat{\mathcal{C}}$ - in accordance with (2.2). According to the general principles of [2], if the mass $m$ of the adjoint matter multiplet is non-vanishing, $\frac{m}{2 \sqrt{2}}$ is the residue of (8.4) at its singularities (which are two simple poles, both located at $x=\infty$, but on different sheets of elliptic curve $\hat{\mathcal{C}}$ ).

The (reduced) prepotential - as given by eq.(6.4) - is

$$
\begin{gathered}
\mathcal{F}_{\tau}(a, m)=\frac{1}{2} a a_{D}+\frac{i m}{2 \sqrt{2}} \int_{8 h_{2} / m^{2}}^{\infty} \sqrt{\frac{\frac{m^{2}}{8} x-h_{2}}{\prod_{a=1}^{3}\left(x-\hat{e}_{a}(\tau)\right)}} d x, \\
a=\frac{2}{\pi} \int_{\hat{e}_{1}}^{\hat{e}_{2}} \sqrt{\frac{h_{2}-\frac{m^{2}}{8} x}{\prod_{a=1}^{3}\left(x-\hat{e}_{a}(\tau)\right)}} d x, \quad a_{D}=\frac{2}{\pi} \int_{\hat{e}_{2}}^{\hat{e}_{3}} \sqrt{\frac{h_{2}-\frac{m^{2}}{8} x}{\prod_{a=1}^{3}\left(x-\hat{e}_{a}(\tau)\right)}} d x .
\end{gathered}
$$

In this formula $\tau$ is a parameter, not an argument of the prepotential. It is of interest also to consider it as one of the arguments, on equal footing with the other moduli. As usual in the presence of massive matter multiplets the integral for the prepotential is logarithmically divergent, the coefficient of the logarithm is just $\frac{m^{2}}{8}$ and is independent of $h_{2}$ or $\tau$. 


\subsection{The $m^{2}=0(N=4$ SUSY $)$ limit}

In this limit $g^{2} \sim m^{2}=0$ and the curve $\mathcal{C}$ degenerates into (two copies) of the bare spectral curve:

$$
\hat{\mathcal{C}} \stackrel{m^{2}=0}{\longrightarrow} E(\tau)
$$

while

$$
d \mathcal{S}_{\min } \cong 2 t d \omega_{0} \stackrel{g^{2}=0}{\longrightarrow} 2 \sqrt{h_{2}} d \omega_{0}
$$

- into the multiple of canonical holomorphic $(1,0)$-differential on $E(\tau)$, given by $(8.2)$.

The periods

$$
a \rightarrow 2 \sqrt{h_{2}}, \quad a_{D} \rightarrow 2 \tau \sqrt{h_{2}},
$$

and the prepotential (8.10) turns into

$$
\mathcal{F}_{\tau}(a)=\frac{1}{2} a a_{D}=\frac{1}{2} a^{2} \tau=2 h \tau .
$$

All this is in accordance with [2].

Description of the $N=4$ SUSY limit $g^{2} \sim m^{2}=0$ is equally simple for any group $S L\left(N_{c}\right)$. Its characteristic feature is that the spectral parameter $z$ disappears from the Lax operator in eq.(6.1) for the spectral curve $\mathcal{C}$ and (6.1) turns into:

$$
P_{N_{c}}(t) \equiv \sum_{l=0}^{N_{c}} s_{l}(h) t^{N_{c}-l}=0,
$$

solutions to which are just $t=t_{\gamma}, \gamma=1, \ldots, N_{c}$, such that $h_{k}=\frac{1}{k} \sum_{\gamma=1}^{N_{c}} t_{\gamma}^{k}$ (and $h_{1}=\sum_{\gamma} t_{\gamma}=0$ for $S L\left(N_{c}\right)$ ). We see that the curve $\mathcal{C}$ splits into $N_{c}$ copies (glued together at the points $t_{\gamma}=t_{\gamma^{\prime}}$, where the Hamiltonians $h_{k}$ become algebraically dependent). The differential

$$
d \mathcal{S}_{\min } \cong \oplus_{\gamma} t_{\gamma} d \omega_{0}(z)
$$

it is free of singularities, thus all the corresponding $T_{n}=0$. Its periods are just

$$
a_{\gamma}=t_{\gamma} \oint_{A_{0}} d \omega_{0}=t_{\gamma}, \quad a_{\gamma}^{D}=t_{\gamma} \oint_{B_{0}} d \omega_{0}=\tau t_{\gamma}
$$

(one can take any $N_{c}-1$ of these for $a_{i}$ and $a_{i}^{D}$ ). The prepotential in this case is

$$
\mathcal{F}_{\text {red }}\left(a_{i}\right)=\frac{1}{2} \sum_{\gamma} a_{\gamma} a_{\gamma}^{D}=\frac{1}{2} \tau \sum_{\gamma} a_{\gamma}^{2},
$$

it is obviously homogeneous of degree 2, since $\tau$ is a parameter of $E(\tau)$, which is by definition independent of $a_{i}$ - and this is in accordance with both conformal invariance of the $N=4$ SUSY theory and with eq.(4.8) when all $T_{n}=0$. 


\subsection{The double scaling $m^{2}=\infty$ (pure gauge $N=2$ or sine-Gordon) limit}

The limit $g^{2} \sim m^{2} \rightarrow \infty$ is more sophisticated. Of interest is actually a double scaling limit $[26,4]$, when also $q=e^{i \pi \tau} \rightarrow 0$, so that dimensional trasmutation takes place and the new massive parameter emerges,

$$
\Lambda^{2}=2 m^{2} q
$$

The scaling rule for $\xi$ is:

$$
\xi=\frac{i w_{1}}{2 \pi} \log \frac{z}{q}, \quad \frac{2}{w_{1}} d \xi=\frac{i}{\pi} \frac{d z}{z}
$$

We have:

$$
\wp(\xi)=\left(\frac{\pi}{i w_{1}}\right)^{2}\left(C(\tau)+4 q\left(z+\frac{1}{z}\right)+o\left(q^{2}\right)\right)
$$

where

$$
\begin{gathered}
C(\tau)=\frac{1}{3} \frac{\partial \log \Delta\left(q^{2}\right)}{\partial \log q^{2}}=\frac{1}{3}\left(1-24 \sum_{M \geq 1} \frac{q^{2 M}}{\left(1-q^{2 M}\right)^{2}}\right)=\frac{1}{3}\left(1-24 q^{2}-72 q^{4}-96 q^{6}-\ldots\right), \\
\Delta\left(q^{2}\right) \equiv \eta^{24}\left(q^{2}\right) \equiv q^{2} \prod_{n \geq 1}\left(1-q^{2 n}\right)^{24}=\frac{9}{256}\left(\hat{e}_{1}-\hat{e}_{2}\right)^{2}\left(\hat{e}_{2}-\hat{e}_{3}\right)^{2}\left(\hat{e}_{3}-\hat{e}_{1}\right)^{2} .
\end{gathered}
$$

Therefore

$$
\begin{aligned}
h_{2}+g^{2} \wp(\xi) & =\left(h_{2}+\frac{m^{2}}{8} C(\tau)\right)+\frac{1}{2} m^{2} q\left(z+\frac{1}{z}\right)+o\left(q^{2}\right) \equiv \\
& \equiv \frac{1}{2}\left(u+\frac{1}{2} \Lambda^{2}\left(z+\frac{1}{z}\right)+o\left(q^{2}\right)\right) .
\end{aligned}
$$

The double scaling limit is the one when $m \rightarrow \infty, q \rightarrow 0$ (i.e. $\tau \rightarrow+i \infty$ ), $h=h^{N=4} \rightarrow \infty$, but $\Lambda^{2}$ and $^{12}$

$$
u \equiv 2 h_{2}^{N=2} \equiv 2\left(h_{2}^{N=4}+\frac{m^{2}}{8} C(\tau)\right)
$$

remain finite. Then

$$
h_{2}+g^{2} \wp(\xi) \stackrel{\text { sc.l. }}{\longrightarrow} \frac{u}{2}+\frac{\Lambda^{2}}{4}\left(z+\frac{1}{z}\right)=\frac{1}{2}(u-\tilde{x}),
$$

where $\frac{2}{w_{1}} d \xi=\tilde{x} \equiv-\frac{\Lambda^{2}}{2}\left(z+\frac{1}{z}\right)$, so that $\frac{i}{\pi} \frac{d z}{z}=\frac{i}{\pi} \frac{d \tilde{x}}{\sqrt{\widetilde{x}^{2}-\Lambda^{4}}}$.

Given all this,

$$
d \mathcal{S}_{\min }=\frac{2}{w_{1}} \sqrt{h_{2}+g^{2} \wp(\xi)} d \xi \stackrel{\text { sc.l. }}{\longrightarrow} \frac{1}{\pi \sqrt{2}} \sqrt{\frac{\tilde{x}-u}{\tilde{x}^{2}-\Lambda^{4}}} d \tilde{x},
$$

in accordance with (7.2) and [1].

\footnotetext{
${ }^{12}$ It deserves noting that $C(\tau) \neq \frac{1}{2} \hat{e}_{1}(\tau)=2 C(2 \tau)-C(\tau)$, and thus (8.25) is different from eq.(16.25) of [2]. See [4] for more details.
} 


\subsection{The double scaling limit in elliptic parametrization}

It is instructive to consider the same limit using elliptic parametrization. This helps to follow degeneration of $\hat{\mathcal{C}}$ into a punctured sphere. As we found in the previous section 8.3 , the coordinate $x$ on $E(\tau)$ in the double scaling limit is substituted by $\tilde{x}$ :

$$
x=\left(\frac{w_{1}}{\pi}\right)^{2} \wp(\xi)=-\left(C(\tau)+4 q\left(z+\frac{1}{z}\right)+o\left(q^{2}\right)\right)=-\frac{1}{3}+\frac{8 q}{\Lambda^{2}} \tilde{x}+o\left(q^{2}\right),
$$

so that $d x=\frac{8 q}{\Lambda^{2}} d \tilde{x}(1+o(q))$. Equation (8.1) for $E(\tau)$ contains also $\hat{e}_{a}(\tau)$, which are expressible in terms of the theta-constants:

$$
\begin{gathered}
\hat{e}_{1}(\tau)=\frac{2 \theta_{00}^{4}-\theta_{10}^{4}}{3}=\frac{2}{3}\left(1+24 q^{2}+24 q^{4}+\ldots\right)=\frac{2}{3}+o\left(q^{2}\right), \\
\hat{e}_{2}(\tau)=-\frac{\theta_{00}^{4}+\theta_{10}^{4}}{3}=-\frac{1}{3}\left(1+24 q+24 q^{2}+96 q^{3}+24 q^{4}+144 q^{5}+\ldots\right)=-\frac{1}{3}-8 q+o\left(q^{2}\right), \\
\hat{e}_{3}(\tau)=\frac{2 \theta_{10}^{4}-\theta_{00}^{4}}{3}=-\frac{1}{3}\left(1-24 q+24 q^{2}-96 q^{3}+24 q^{4}-144 q^{5}+\ldots\right)=-\frac{1}{3}+8 q+o\left(q^{2}\right)
\end{gathered}
$$

Thus $E(\tau)$ becomes:

$$
y^{2}=\prod_{a=1}^{3}\left(x-\hat{e}_{a}(\tau)\right)=-\frac{64 q^{2}}{\Lambda^{4}}\left(\tilde{x}^{2}-\Lambda^{4}\right)(1+o(q))
$$

and

$$
\frac{d x}{y(x)}=-i \frac{d \tilde{x}}{\sqrt{\tilde{x}^{2}-\Lambda^{4}}}(1+o(q)) .
$$

When multiplied by $\frac{1}{\pi} \sqrt{h-\frac{m^{2}}{8} x}=\frac{i}{\sqrt{2} \pi} \sqrt{\tilde{x}-u}(1+o(q))$, this once again reproduces (7.2).

\subsection{Picard-Fuchs equations for $\oint d \mathcal{S}_{\min }$}

Let us denote

$$
<(\ldots)>\equiv \oint d x \sqrt{\frac{\hat{h}-x}{\prod_{a=1}^{3}\left(x-\hat{e}_{a}(\tau)\right)}}(\ldots)=\oint d \hat{\mathcal{S}}_{\min }(\ldots) .
$$

Then $a$ and $a^{D}$ are given by $\frac{m}{2 \sqrt{2} \pi}<1>$, and $\hat{h} \equiv \frac{8 h_{2}}{m^{2}}, d \hat{\mathcal{S}}_{\text {min }} \equiv \frac{2 \sqrt{2} \pi}{m} d \mathcal{S}_{\min }$. The Ward identity:

$$
<\left(\frac{1}{\hat{h}-x}+\sum_{a=1}^{3} \frac{1}{x-\hat{e}_{a}}\right) \delta x>=2<\frac{d \delta x}{d x}>
$$


holds for any $\delta x$. Its particular examples are:

$$
\begin{aligned}
\delta x=1: & <\left(\frac{1}{\hat{h}-x}+\sum_{a=1}^{3} \frac{1}{x-\hat{e}_{a}}\right)>=0 \\
\delta x=x: & <\left(\frac{\hat{h}}{\hat{h}-x}+\sum_{a=1}^{3} \frac{\hat{e}_{a}}{x-\hat{e}_{a}}\right)>=0 \\
\delta x=\frac{1}{\hat{h}-x}: \quad & <\frac{1}{(\hat{h}-x)^{2}}>=\sum_{a=1}^{3}<\frac{1}{\left(x-\hat{e}_{a}\right)(\hat{h}-x)}>= \\
& =\left(\sum_{a=1}^{3} \frac{1}{\hat{h}-\hat{e}_{a}}\right)<\frac{1}{\hat{h}-x}>+\sum_{a=1}^{3}\left(\frac{1}{\hat{h}^{-\hat{e}_{a}}}<\frac{1}{x-\hat{e}_{a}}>\right)
\end{aligned}
$$

Combining the first and the third of these relations we get:

$$
\begin{gathered}
<\frac{1}{(\hat{h}-x)^{2}}>-\frac{2}{3}\left(\sum_{b=1}^{3} \frac{1}{\hat{h}-\hat{e}_{b}}\right)<\frac{1}{\hat{h}-x}>=\sum_{a=1}^{3}<\frac{E_{a}}{x-\hat{e}_{a}}>, \\
E_{a}(\hat{h} \mid \tau) \equiv \frac{1}{\hat{h}-\hat{e}_{a}}-\frac{1}{3}\left(\sum_{b=1}^{3} \frac{1}{\hat{h}-\hat{e}_{b}}\right), \quad \sum_{a=1}^{3} E_{a}=0 .
\end{gathered}
$$

This list should be supplemented by $\left(q=e^{i \pi \tau}\right)$ :

$$
\frac{\partial}{\partial \log q}<1>=\frac{1}{2} \sum_{a=1}^{3}<\frac{\partial \hat{e}_{a} / \partial \log q}{x-\hat{e}_{a}}>
$$

Relation (it can be considered as a definition of $f(\hat{h} \mid \tau)$ and $g(\hat{h} \mid \tau)$, see also (8.41) below)

$$
\frac{\partial \hat{e}_{a}(\tau)}{\partial \log q}=f(\hat{h} \mid \tau) \hat{e}_{a}(\tau)+g(\hat{h} \mid \tau) E_{a}(\hat{h} \mid \tau)
$$

implies the Picard Fuchs equation for $d \mathcal{S}_{\min }$ in the form:

$$
\left(\frac{\partial}{\partial \log q}+\hat{h} f(\hat{h} \mid \tau) \frac{\partial}{\partial \hat{h}}+2 g(\hat{h} \mid \tau)\left[\frac{\partial^{2}}{\partial \hat{h}^{2}}+\frac{1}{3}\left(\sum_{b=1}^{3} \frac{1}{\hat{h}-\hat{e}_{b}}\right) \frac{\partial}{\partial \hat{h}}\right]\right) d \hat{\mathcal{S}}_{\min } \cong 0 .
$$

Now we need to find $f(\hat{h} \mid \tau)$ and $g(\hat{h} \mid \tau)$ from (8.34). Of these three equations only two are independent, since the sum of the three is zero. We begin from rewriting $E_{a}$ in a more convenient form:

$E_{a}=\frac{3 \hat{h} \hat{e}_{a}+\hat{e}_{a}^{2}+2 \varepsilon_{a b c} \hat{e}_{b} \hat{e}_{c}}{3 y^{2}(\hat{h})}=\frac{\hat{h} \hat{e}_{a}+\hat{e}_{a}^{2}-\frac{1}{3} \sum_{b=1}^{3} \hat{e}_{b}^{2}}{y^{2}(\hat{h})}, \quad \hat{e}_{a} E_{b}-\hat{e}_{b} E_{a}=-\frac{\left(\hat{e}_{a}-\hat{e}_{b}\right)\left(\hat{e}_{a} \hat{e}_{b}+2 \varepsilon_{a b c} \hat{e}_{c}^{2}\right)}{3 y^{2}(\hat{h})}$.

Here $y^{2}(\hat{h})=\prod_{a=1}^{3}\left(\hat{h}-\hat{e}_{a}(\tau)\right)$, and $\varepsilon_{123}=\varepsilon_{213}=\ldots=1, \varepsilon_{112}=\ldots=0$, with no summation over repeated indices. Using these formulas we obtain from (8.34):

$$
\begin{gathered}
g(\hat{h} \mid \tau)=\frac{-\frac{\partial \hat{e}_{1}}{\partial \log q} \hat{e}_{2}+\frac{\partial \hat{e}_{2}}{\partial \log q} \hat{e}_{1}}{\hat{e}_{1} E_{2}-\hat{e}_{2} E_{1}}=3 y^{2}(\hat{h}) \hat{g}=-y^{2}(\hat{h}), \\
f(\hat{h} \mid \tau)=\frac{\frac{\partial \hat{e}_{1}}{\partial \log q} E_{2}-\frac{\partial \hat{e}_{2}}{\partial \log q} E_{1}}{\hat{e}_{1} E_{2}-\hat{e}_{2} E_{1}}=-(3 \hat{h} \hat{g}+\hat{f})=\hat{h}+C(\tau) .
\end{gathered}
$$


In more detail, the coefficients at the r.h.s. are:

$$
\begin{gathered}
\hat{g} \equiv \frac{\frac{\partial \hat{e}_{1}}{\partial \log q} \hat{e}_{2}-\frac{\partial \hat{e}_{2}}{\partial \log q} \hat{e}_{1}}{\left(\hat{e}_{1}-\hat{e}_{2}\right)\left(\hat{e}_{1} \hat{e}_{2}+2 \hat{e}_{3}^{2}\right)}=\frac{\frac{16}{3} q\left(1-12 q^{2}+54 q^{4}-\ldots\right)}{-16 q\left(1-12 q^{2}+54 q^{4}-\ldots\right)}=-\frac{1}{3}, \\
\hat{f} \equiv \frac{\frac{\partial \hat{e}_{1}}{\partial \log q}\left(2 \hat{e}_{1} \hat{e}_{3}+\hat{e}_{2}^{2}\right)-\frac{\partial \hat{e}_{2}}{\partial \log q}\left(2 \hat{e}_{2} \hat{e}_{3}+\hat{e}_{1}^{2}\right)}{\left(\hat{e}_{1}-\hat{e}_{2}\right)\left(\hat{e}_{1} \hat{e}_{2}+2 \hat{e}_{3}^{2}\right)}=\frac{\frac{16}{3} q\left(1-36 q^{2}+270 q^{4}-\ldots\right)}{-16 q\left(1-12 q^{2}+54 q^{4}-\ldots\right)}= \\
=-\frac{1}{3}\left(1-24 q^{2}-72 q^{4}-\ldots\right)=-C(\tau)=-\frac{1}{3} \frac{\partial \log \Delta\left(q^{2}\right)}{\partial \log q^{2}} .
\end{gathered}
$$

Note that $C(\tau)$ here is exactly the same as in (8.22).

As additional check, one can rewrite (8.34) as

$$
\frac{\partial \log \theta_{00}^{4}}{\partial \log q}=\frac{\partial \log \left(\hat{e}_{1}-\hat{e}_{2}\right)}{\partial \log q}=f(\hat{h} \mid \tau)+g(\hat{h} \mid \tau) \frac{h-\hat{e}_{3}}{y^{2}(\hat{h})}
$$

plus two analogous relations for $\theta_{10}$ and $\theta_{01}$. Adding all the three one gets,

$$
f(\hat{h} \mid \tau)+\frac{h g(\hat{h} \mid \tau)}{y^{2}(\hat{h})}=\frac{1}{3} \frac{\partial \log \left(\theta_{00}^{4} \theta_{10}^{4} \theta_{01}^{4}\right)}{\partial \log q}=\frac{1}{3} \frac{\partial \log \Delta\left(q^{2}\right)}{\partial \log q^{2}}=C(\tau),
$$

(since $\left.\Delta\left(q^{2}\right)=\left(\theta_{00} \theta_{10} \theta_{01}\right)^{8}=\left(\theta_{11}^{\prime}\right)^{8}\right)$. Another linear combination of formulas (8.38) gives:

$$
g(\hat{h} \mid \tau)=\frac{y^{2}(\hat{h})}{\theta_{01}^{4}} \frac{\partial \log \left(\theta_{00}^{4} / \theta_{10}^{4}\right)}{\partial \log q}=-y^{2}(\hat{h}) .
$$

Of course, when (8.36) are substituted into (8.34), the $\hat{h}$-dependence cancels at the r.h.s.:

$$
\frac{\partial \hat{e}_{a}}{\partial \log q}=\frac{\mathrm{g}_{2}}{6}+C \hat{e}_{a}-\hat{e}_{a}^{2}
$$

where

$$
\begin{gathered}
\mathrm{g}_{2}(\tau)=-4\left(\hat{e}_{1} \hat{e}_{2}+\hat{e}_{2} \hat{e}_{3}+\hat{e}_{3} \hat{e}_{1}\right)=2 \sum_{a=1}^{3} \hat{e}_{a}^{2}=\frac{2}{3}\left(\theta_{00}^{8}+\theta_{01}^{8}+\theta_{10}^{8}\right)= \\
=\frac{4}{3}\left(1+240\left(q^{2}+9 q^{4}+28 q^{6}+\ldots\right)\right)=\frac{4}{3}\left(1+240 q^{2}+2160 q^{4}+6720 q^{6}+\ldots\right) .
\end{gathered}
$$

Substituting (8.36) into (8.35), we obtain

$$
\frac{1}{2} \frac{\partial}{\partial \log q} d \hat{\mathcal{S}}_{\min } \cong\left(y^{2}(\hat{h}) \frac{\partial^{2}}{\partial \hat{h}^{2}}+\left[\frac{1}{2} \hat{h}^{2}-\frac{1}{2} \hat{h} C(\tau)-\frac{1}{12} \mathrm{~g}_{2}(\tau)\right] \frac{\partial}{\partial \hat{h}}\right) d \hat{\mathcal{S}}_{\min }
$$

\subsection{The $m^{2} \rightarrow \infty, q \rightarrow 0$ double scaling $(N=2)$ limit of Picard-Fuchs equation}

We first consider the double scaling limit, $m^{2} \rightarrow \infty, q \rightarrow 0$. For small $q$ the operator at the r.h.s. of (8.43) becomes:

$$
\left(\left(\hat{h}+\frac{1}{3}\right)^{2}-8 q^{2}\right)\left(\hat{h}-\frac{2}{3}\right) \frac{\partial^{2}}{\partial \hat{h}^{2}}+\frac{1}{2}\left(\hat{h}+\frac{1}{3}\right)\left(\hat{h}-\frac{2}{3}\right) \frac{\partial}{\partial \hat{h}}
$$


(one can use the above-listed asymptotic expansions for $\hat{e}_{a}(\tau)$ and $C(\tau)$ ). According to (8.25) and (8.19) these are $\hat{h} \equiv \frac{8 h}{m^{2}}, u$ and $\Lambda$, that are finite in the double scaling limit, and

$$
\hat{h}+\frac{1}{3}=\hat{h}+C(\tau)+o\left(q^{2}\right)=\frac{4 u}{m^{2}}+o\left(q^{2}\right)=\frac{8 u}{\Lambda^{2}} q+o\left(q^{2}\right) .
$$

After this substitution (8.44) becomes:

$$
\left(\Lambda^{4}-u^{2}\right) \frac{\partial^{2}}{\partial u^{2}}-\frac{1}{2} u \frac{\partial}{\partial u}
$$

Now, turn to the 1.h.s. of (8.43). Obviously,

$$
\left.\frac{1}{2} \frac{\partial}{\partial \log q}\right|_{h=\text { const }}=\frac{1}{2}\left(\left.\frac{\partial}{\partial \log q}\right|_{u=\text { const }}-\left[\hat{h}+\frac{1}{3}\right] \frac{\partial}{\partial \hat{h}}\right)=\left.\frac{1}{2} \frac{\partial}{\partial \log q}\right|_{u=\text { const }}-\frac{1}{2} u \frac{\partial}{\partial u} .
$$

The second item at the r.h.s. is exactly what necessary to cancel the one at the r.h.s. of (8.46). The $q$-derivative should be irrelevant in the limit, when it acts on $d \mathcal{S}_{\min }$, but (8.43) is written for $d \hat{\mathcal{S}}_{\text {min }} \sim \frac{1}{m} d \mathcal{S}_{\text {min }} \sim q^{1 / 2} d \mathcal{S}_{\text {min }}$. Thus we should substitute $\frac{1}{2} \frac{\partial}{\partial \log q} d \hat{\mathcal{S}}_{\text {min }} \rightarrow \frac{1}{4} d \hat{\mathcal{S}}_{\text {min }}$. Putting all together we obtain as the double-scaling limit of eq.(8.43):

$$
\left(\left(u^{2}-\Lambda^{4}\right) \frac{\partial^{2}}{\partial u^{2}}+\frac{1}{4}\right) d \mathcal{S}_{\min } \cong 0
$$

exactly the right Picard-Fuchs equation (7.12).

\subsection{The $m^{2}=0(N=4)$ limit}

Proceed now to another limit, when $m^{2}=0$. In the first approximation, we can just say that $\hat{h} \equiv \frac{8 h}{m^{2}}$ is larger than everything in this limit and (8.43) reduces to:

$$
\left(\hat{h}^{3} \frac{\partial^{2}}{\partial \hat{h}^{2}}+\frac{1}{2} \hat{h}^{2} \frac{\partial}{\partial \hat{h}}\right) d \mathcal{S}_{\min } \cong 0
$$

what gives the answer $d \mathcal{S}_{\min } \sim \sqrt{\hat{h}}$ - in consistency with (8.12), $d \mathcal{S}_{\min } \cong 2 \sqrt{h} d \omega_{0}$. This accuracy, however, is not enough to determine the $\tau$ dependence of the periods ( 1 and $\tau$ ) of $d \omega_{0}$. Moreover, one can wonder how two such different periods can satisfy the same equation. Resolution to this puzzle is simple: to get the next approximation we restore $m^{2}$-dependence in (8.43) and substitute $d \mathcal{S}_{\min }=2 \sqrt{h_{2}} d S_{0}(\tau)-\frac{m^{2}}{8 \sqrt{h_{2}}} d S_{1}(\tau):$

$$
\left(\frac{\partial}{\partial \log q}+\frac{1}{2} C(\tau)\right) d S_{0}(\tau) \cong-\frac{1}{2} d S_{1}(\tau),
$$

and this is not a closed equation for $d S_{0}(\tau)$ alone.

One can check that $d S_{0}=d \omega_{0}$ and $d S_{1}=x d \omega_{0}$ - as implied by $\frac{1}{h_{2}}$-expansion of $d \mathcal{S}_{\min }(8.9)$ - do indeed satisfy (8.50). This follows from the Ward identities for the average

$$
\langle\langle(\ldots)\rangle\rangle \equiv \oint d \omega_{0}(\ldots)=\frac{1}{2 \pi} \oint \frac{d x}{\sqrt{\prod_{a=1}^{3}\left(x-\hat{e}_{a}\right)}}(\ldots),
$$




$$
\begin{array}{ll}
\delta x=1: & \sum_{a=1}^{3}\left\langle\left\langle\frac{1}{x-\hat{e}_{a}}\right\rangle\right\rangle=0, \\
\delta x=x: \quad & \sum_{a=1}^{3}\left\langle\left\langle\frac{\hat{e}_{a}}{x-\hat{e}_{a}}\right\rangle\right\rangle=-\langle\langle 1\rangle\rangle, \\
\delta x=x^{2}: & \sum_{a=1}^{3}\left\langle\left\langle\frac{\hat{e}_{a}^{2}}{x-\hat{e}_{a}}\right\rangle\right\rangle=\langle\langle x\rangle\rangle,
\end{array}
$$

together with (8.41):

$$
\begin{gathered}
\frac{\partial}{\partial \log q} \oint d \omega_{0}=\frac{1}{2} \sum_{a=1}^{3}\left\langle\left\langle\frac{\partial \hat{e}_{a} / \partial \log q}{x-\hat{e}_{a}}\right\rangle\right\rangle \stackrel{(8.41)}{=} \\
=\frac{\mathrm{g}_{2}}{12} \sum_{a=1}^{3}\left\langle\left\langle\frac{1}{x-\hat{e}_{a}}\right\rangle\right\rangle+\frac{C(\tau)}{2} \sum_{a=1}^{3}\left\langle\left\langle\frac{\hat{e}_{a}}{x-\hat{e}_{a}}\right\rangle\right\rangle-\frac{1}{2} \sum_{a=1}^{3}\left\langle\left\langle\frac{\hat{e}_{a}^{2}}{x-\hat{e}_{a}}\right\rangle\right\rangle \stackrel{(8.51)}{=} \\
=-\frac{1}{2}\langle\langle x\rangle\rangle-\frac{C(\tau)}{2}\langle\langle 1\rangle\rangle=-\frac{1}{2} \oint x d \omega_{0}-\frac{C(\tau)}{2} \oint d \omega_{0},
\end{gathered}
$$

i.e. indeed, as required by (8.50), for any contour

$$
\left(\frac{\partial}{\partial \log q}+\frac{1}{2} C(\tau)\right) \oint d \omega_{0}=-\frac{1}{2} \oint x d \omega_{0}
$$

This identity can be also reinterpreted as the vanishing of residues of Weierstrass function, so that $\zeta$-function, $\zeta(\xi)=-\int^{\xi} \wp(\xi) d \xi$, locally exists, and

$$
\begin{gathered}
\oint_{A} x d \omega_{0}=\frac{w_{1}}{\pi^{2}} \int_{0}^{w_{1}} \wp(\xi) d \xi=\frac{w_{1}}{\pi^{2}}\left(\zeta(\xi)-\zeta\left(\xi+2 w_{1}\right)\right) \equiv-\frac{2 w_{1} \eta_{1}}{\pi^{2}}= \\
=-\left(C(\tau)+2 \frac{\partial}{\partial \log q}\right) \oint_{A} d \omega_{0}=-C(\tau), \quad \oint_{B} x d \omega_{0}=-\frac{2 w_{1} \eta_{2}}{\pi^{2}}=-\frac{2}{i \pi}-\tau C(\tau) .
\end{gathered}
$$

It deserves noting, that eq.(8.50) and thus (8.43) has many other solutions, for example:

$$
d \mathcal{S}_{\min }\left(1+o\left(h_{2}^{-2}\right)\right) d S_{0} \sim \Delta^{-1 / 12}\left(q^{2}\right) \sqrt{h_{2}}=\frac{\sqrt{h_{2}}}{\operatorname{Det} \bar{\partial}_{0}}, \quad d S_{1}=0 .
$$

Such extra solutions are eliminated by the second Picard-Fuchs equation, which is not considered in this paper.

\subsection{Canonical form of the Picard-Fuchs equation for the case of elliptic bare spectral curve}

The form of the r.h.s. of (8.43) implies that the "proper" variable in it is not $\hat{h}$, but rather $\chi$, such that $d \chi \sim d \hat{h} / y(\hat{h})$, or $\hat{h}=\wp(\chi)$. More precisely, one can perform a conjugation, $d \mathcal{S}_{\min }=U(\hat{h}, \tau) d \check{\mathcal{S}}$ 
and convert (8.43) into:

$$
\frac{1}{2} \frac{\mathcal{D}}{\mathcal{D} \log q} d \check{\mathcal{S}} \cong\left[\left(y(\hat{h}) \frac{\partial}{\partial \hat{h}}\right)^{2}+V(\hat{h}, \tau)\right] d \check{\mathcal{S}} .
$$

We are not aware of exact form of the conjugation trasform and thus of $V(\hat{h}, \tau)$ in $(8.56)$, thus we present only some preliminary formulas.

The most reasonable candidate for the role of $U(\hat{h}, \tau)$ seems to be

$$
U(\hat{h}, \tau)=(\hat{h}-2 C(\tau))^{1 / 8} y^{1 / 4}(\hat{h})=\left[(\hat{h}-2 C(\tau)) \prod_{a=1}^{3}\left(\hat{h}-\hat{e}_{a}(\tau)\right)\right]^{1 / 8} .
$$

After such conjugation the $\partial / \partial \hat{h}$-term in (8.43) is not completely eliminated - in order to give the r.h.s. of (8.56),- but turns into

$$
\begin{gathered}
\left(\frac{\hat{h}^{2}}{2}-\frac{C \hat{h}}{2}-\frac{\mathrm{g}_{2}}{12}+2 y^{2}(\hat{h}) \frac{\partial \log U}{\partial \hat{h}}-\frac{\partial \log y(\hat{h})}{\partial \hat{h}}\right) \frac{\partial}{\partial \hat{h}}= \\
=\left(\frac{1}{4} y^{2}(\hat{h})\left(\frac{1}{\hat{h}-2 C}+\sum_{a=1}^{3} \frac{1}{\hat{h}-\hat{e}_{a}}\right)-\left(\hat{h}^{2}+\frac{C \hat{h}}{2}-\frac{\mathrm{g}_{2}}{24}\right)\right) \frac{\partial}{\partial \hat{h}}= \\
=\frac{1}{4}\left(\frac{\hat{h}^{3}-\frac{1}{4} \mathrm{~g}_{2} \hat{h}-\frac{1}{4} \mathrm{~g}_{3}}{\hat{h}-2 C}-\left(\hat{h}^{2}+2 C \hat{h}+\frac{\mathrm{g}_{2}}{12}\right)\right) \frac{\partial}{\partial \hat{h}}
\end{gathered}
$$

What is special about this sophisticated expression is that its $\tau$-independent part vanishes. To be precise, near $q=0$ the r.h.s. of (8.58) becomes

$$
\left(\frac{\left(\hat{h}+\frac{1}{3}\right)^{2}\left(\hat{h}-\frac{2}{3}\right)}{\hat{h}-\frac{2}{3}}-\left(\hat{h}^{2}+\frac{2}{3} \hat{h}+\frac{1}{9}\right)+o\left(q^{2}\right)\right) \frac{\partial}{\partial \hat{h}}=o\left(q^{2}\right) \frac{\partial}{\partial \hat{h}} .
$$

This, in turn, implies that such term can be absorbed into the l.h.s. of (8.43) by the following trick. In (8.43) the $(\log q)$-derivative is taken at constant $\hat{h}$. Imagine now that we instead keep constant some other variable, $H=H(\hat{h}, \tau)$ (ideally $H \sim \chi$, but this is not guaranteed by our reasoning). Then

$$
\left.\frac{\mathcal{D}}{\mathcal{D} \log q} \equiv \frac{\partial}{\partial \log q}\right|_{H=\text { const }}=\left.\frac{\partial}{\partial \log q}\right|_{\hat{h}}+\frac{\partial H}{\partial \log q} \frac{\partial}{\partial H}=\frac{\partial}{\partial \log q}+\frac{\partial H}{\partial \log q} \frac{\partial \hat{h}}{\partial H} \frac{\partial}{\partial \hat{h}} .
$$

Specific feature of such correction is that (as long as $H$ is expandable in powers of $q$ ) it vanishes at $q=0$ - and this is exactly what (8.58) does.

One can proceed a little further, considering $\frac{1}{\hat{h}}$-expansion of $(8.58)$ - though we restrict our consideration to the first non-trivial term of this expansion. The r.h.s. of (8.58) is actually:

$$
\left[\left(C^{2}(\tau)-\frac{\mathrm{g}_{2}(\tau)}{12}\right)+o\left(\frac{1}{\hat{h}}\right)\right] \frac{\partial}{\partial \hat{h}} .
$$


Of course, $C^{2}-\frac{1}{12} \mathrm{~g}_{2}=o\left(q^{2}\right)$, as all the other coefficients in the $\hat{h}^{-1}$-expansion. Moreover, it is easy to check that

$$
C^{2}-\frac{\mathrm{g}_{2}}{12}=2 \frac{\partial C}{\partial \log q}
$$

so that

$$
\frac{\partial H}{\partial \log q} \frac{\partial \hat{h}}{\partial H}=-4 \frac{\partial C}{\partial \log q}+o\left(\frac{1}{\hat{h}}\right)
$$

and $H \sim e^{-4 C(\tau)} \hat{h}^{r}\left(\right.$ const $\left.+o\left(\hat{h}^{-1}\right)\right)$ (the value of $r$ does not affect our reasoning, if $H \sim e^{-4 C(\tau)} \chi$ we need $r=-\frac{1}{2}$ ).

Finally, to get some impression of what $V(\hat{h})$ in (8.56) can look like, evaluate it with our $U(\hat{h})=\left(\hat{h}-\frac{2}{3}\right)^{1 / 4}\left(\hat{h}+\frac{1}{3}\right)^{1 / 4}+o\left(q^{2}\right)$ at $q=0$. The answer is:

$$
\begin{gathered}
V(\hat{h})=U^{-1}\left[\left(\hat{h}+\frac{1}{3}\right)^{2}\left(\hat{h}-\frac{2}{3}\right) \frac{\partial^{2}}{\partial \hat{h}^{2}}+\frac{1}{2}\left(\hat{h}+\frac{1}{3}\right)\left(\hat{h}-\frac{2}{3}\right) \frac{\partial}{\partial \hat{h}}+o\left(q^{2}\right)\right] U= \\
=-\frac{1}{8} \frac{\hat{h}+\frac{5}{6}}{\hat{h}-\frac{2}{3}}+o\left(q^{2}\right) .
\end{gathered}
$$

This formula implies that one should actually perform a rational transformation $\hat{h} \rightarrow \breve{h}=-\frac{1}{8} \frac{\hat{h}+\frac{5}{6}}{h-\frac{2}{3}}+$ $o\left(q^{2}\right) ; \quad y(\hat{h}) \rightarrow \check{y}(\check{h})$ before switching to the new variables like $\chi$ or $H$ (rational transformation preserve the shape of the vector field $y(\hat{h}) \partial / \partial \hat{h})$.

To summarize, hypothetically, in appropriate coordinates (8.43) acquires "canonical" form:

$$
\frac{1}{2} \frac{\mathcal{D}}{\mathcal{D} \log q} d \check{\mathcal{S}} \cong\left(\left(\check{y}(\check{h}) \frac{\partial}{\partial \check{h}}\right)^{2}+\check{h}\right) d \check{\mathcal{S}}=\left(\frac{\partial^{2}}{\partial \chi^{2}}-g^{2} \wp(\chi)\right) d \check{\mathcal{S}} .
$$

\subsection{Comments}

A few more comments are now in order about the Picard-Fuchs eq.(8.43).

First of all, since we are now in the situation with two moduli, the single equation is not enough to fix the cohomology class of $d \mathcal{S}_{\min }$ unambiguosly: another one is also required. It is not a big problem, but it should be kept in mind in the future work on this subject.

Second, somewhat similar equations were recently introduced in [27]. This whole subject is of course intimately related to the theory of Knizhnik-Zamolodchikov equation.

Third, the appearence of (8.43) is reminiscent of the Schroedinger equation, especially if it can indeed be brought to the form like (8.65): then it is just the Schroedinger equation for $1 d$ Calogero model. Therefore, if successful, the derivation of (8.65) could serve as an illustration of the general principle that the Witham method is essentially the same as quantization - but with 
considerable change in the nature of variables: quantized model lives on the moduli space (the one of "zero-modes" or "collective coordinates"), not on original configuration space.

Forth, if (8.43) can indeed be converted into eq.(8.65), this would allow one to interpret the modulus $\hat{h}$ as belonging to the bare spectral surface $E(\tau)$ (while in the original Seiberg-Witten/sineGordon setting it rather belonged to a fundamental domain of $\Gamma_{2}$ on the upper half-plane). In other words, the full moduli space $\{\hat{h}, \tau\}$ at every given $\tau$ reduces to elliptic curve $E(\tau)$.

These remarks are already enough to explain why eq.(8.43) (and its analogues for other groups) should be investigated more deeply. We are going now to add one more direction: it should be compared to Picard-Fuchs equation for the relevant Calabi-Yau manifold - and this will raise even more puzzles.

\section{Calabi-Yau manifold with $h_{21}=2$ as a spectral hypersur- face}

\subsection{Formulation of the problem}

In this section we briefly consider the simplest application of the prepotential theory - in the version of section 5 - to families of Calabi-Yau manifolds $M(d=3)$. The role of $\Omega$ is played by the holomprphic (3,0)-form, whose existence is peculiar to Calabi-Yau geometry. Requirement (i) of s. 5 is fulfilled if the moduli are introduced in such a way, that derivatives of $\Omega$ over them produce singularities on submanifolds of codimension greater than one. Requirement (ii) is fulfilled, since the number of moduli, $h_{30}(M)+h_{21}(M)$ is the same as $\frac{1}{2} \operatorname{dim} H^{3}(M)$. The modulus, associated with $h_{30}(M)=1$ corresponds to the overall rescaling of $\Omega$ and is not very interesting.

The simplest example of Calabi-Yau family, which is associated with the $S U(2)$ Seiberg-Witten theory $[13,28]$ has two non-trivial moduli: $h_{21}=2 .{ }^{13}$ It results from factorization of the $W P_{1,1,2,2,6}^{12}$ submanifold, described by the equation:

$$
p(z \mid \phi, \psi) \equiv \frac{z_{1}^{12}}{12}+\frac{z_{2}^{12}}{12}+\frac{z_{3}^{6}}{6}+\frac{z_{4}^{6}}{6}+\frac{z_{5}^{2}}{2}+\frac{\phi z_{1}^{6} z_{2}^{6}}{6}+\psi z_{1} z_{2} z_{3} z_{4} z_{5}=0 .
$$

The two moduli are $\phi$ and $\psi$. Relation to $N=2$ SUSY $S U(2)$ pure gauge theory is originally motivated through the arguments like stringy $S$-duality, with unbroken gauge group $S U(2)$ emerging

\footnotetext{
${ }^{13} h_{21}=\operatorname{rank}_{G}+1$, in our case $G=S L(2)$. The unit difference between $h_{21}$ and $\operatorname{rank}_{G}$ corresponds to the dilaton field in the target-space language and to the $\tau$-parameter (modulus of the bare spectral curve) in Whitham terminology.
} 
at the "conifold locus" in the moduli space,

$$
\left(1-\frac{\phi}{i \psi^{6}}\right)^{2}+\frac{1}{\psi^{12}}=0, \quad \text { or } \quad\left(\phi-i \psi^{6}\right)^{2}=1
$$

The periods of $\Omega$ are given by the standard formula: ${ }^{14}$

$$
\Pi_{C}(\phi, \psi) \equiv \oint_{C} d z_{1} d z_{2} d z_{3} d z_{4} d z_{5} e^{p(z \mid \phi, \psi)} .
$$

Our task could be to demonstrate that the periods $\Pi_{C}(\phi, \psi)$ satisfy the same Picard-Fuchs equation (8.43) that the $S L(2)$ Calogero model - what would allow to conclude that the prepotential of the stringy Calabi-Yau model and that of the simple $1 d$ Calogero system are essentially the same. Instead we obtain a very similar, but essentially different equation - as one gets beyond the conifold (double scaling) limit. Elimination or interpretation of this difference remains for future investigations.

\subsection{Vicinity of the conifold locus: the sine-Gordon limit of Calogero model}

The double scaling limit in terms of $\phi$ and $\psi$ is: $\phi, \psi \rightarrow \infty$ so that $\phi-i \psi^{6}$ remains finite. The correspondence between Calabi-Yau model in this limit and original Seiberg-Witten example was already established in [13]. Near the conifold singularity, the most interesting of the periods (9.3) can be reduced (by rescaling of variables and making a saddle-point calculation) to the two-fold integrals

$$
\tilde{\Pi}_{C}\left(\frac{u}{\Lambda^{2}}\right)=\int \frac{d z_{1} d z_{2}}{\left(z_{1} z_{2}\right)^{4}} \exp \left(\frac{z_{1}^{12}}{12}+\frac{z_{2}^{12}}{12}+\frac{u z_{1}^{6} z_{2}^{6}}{6 \Lambda^{2}}\right) .
$$

Only peculiar combination of moduli,

$$
\tilde{u} \equiv \frac{u}{\Lambda^{2}}=\phi-i \psi^{6}
$$

which defines deviation from the conifold locus, survives in this limit. (The locus itself is described as $u^{2}=\Lambda^{4}$.) We refer to [13] for details of the saddle-point calculation, leading from (9.3) to (9.4). For completeness of our presentation and as a warm-up for the similar computation beyond conifold limit, we derive here the Picard-Fuchs equations for $\tilde{\Pi}(\tilde{u})$ and show that they indeed coincide with those for the sine-Gordon model - i.e. with eq.(7.12).

\footnotetext{
14 The flat measure restricted to the hypersurface $p=0$ is $d z_{1} \wedge \ldots \wedge d z_{5} \delta(p(z))=d z_{1} \wedge \ldots \wedge d z_{5} \int d \lambda e^{i \lambda p(z)}$, and the (quasi)homogeneity of of $p(z)$ allows one to eliminate $i \lambda$ in the exponent.
} 
Denote integration in (9.4) with the exponential weight $e^{p(z)}$ by $<\ldots>$, in particular, ${ }^{15} \tilde{\Pi}(\tilde{u})=$ $<\left(z_{1} z_{2}\right)^{-4}>$. There always is a Ward-identity, resulting from the change of integration variables $z_{j}:$

$$
<F(z) \frac{\partial p(z)}{\partial z_{j}} \delta z_{j}+F(z) \frac{\partial \delta z_{j}}{\partial z_{j}}+\frac{\partial F}{\partial z_{j}} \delta z_{j}>=0 .
$$

for any function $F(z)$. Whenever $\delta z_{j}$ is adjusted in such a way that the operator at the l.h.s. can be obtained by action of some differential operator on $\left\langle\left(z_{1} z_{2}\right)^{-4}>\right.$ - we get a Picard-Fuchs equation. In the case of (9.4) the simplest option is

$$
\begin{gathered}
\delta z_{1}=z_{1} z_{2}^{12}, \\
\delta z_{2}=\left(3-\tilde{u}\left(z_{1} z_{2}\right)^{6}\right) z_{2}
\end{gathered}
$$

$\left(\delta z_{2}\right.$ is adjusted to cancel all the terms associated with $\delta z_{1}$, which are not integer powers of $\left.z_{1}^{6} z_{2}^{6}\right)$. With this choice and with $F(z)=\left(z_{1} z_{2}\right)^{-4}$ (9.6) turns into

$$
0=<\left(1-\tilde{u}^{2}\right) z_{1}^{12} z_{2}^{12}-9>=\left(36\left(\Lambda^{4}-u^{2}\right) \frac{\partial^{2}}{\partial u^{2}}-9\right) \tilde{\Pi}\left(\frac{u}{\Lambda^{2}}\right)
$$

what is exactly the Picard-Fuchs equation (7.12) for the sine-Gordon model.

\subsection{Beyond conifold limit}

We can now apply the same trick to derive the Picard-Fuchs equation for $\Pi_{C}(\phi, \psi)$ beyond conifold limit. Now we use $\langle\ldots\rangle$ to denote integration in $(9.3)$, so that $\Pi(\phi, \psi)=<1>$. This time we substitute into $(9.6) F(z)=1$ and

$$
\begin{gathered}
\delta z_{1}=z_{1} z_{2}^{12} \\
\delta z_{2}=-\left(1+\phi\left(z_{1} z_{2}\right)^{6}+\psi z_{1} z_{2} z_{3} z_{4} z_{5}\right) z_{2}
\end{gathered}
$$

and obtain:

$$
\begin{gathered}
0=<\left(1-\phi^{2}\right) z_{1}^{12} z_{2}^{12}-8 \phi z_{1}^{6} z_{2}^{6}-1 \\
-2 \phi z_{1}^{6} z_{2}^{6} \cdot \psi z_{1} z_{2} z_{3} z_{4} z_{5}-3 \psi z_{1} z_{2} z_{3} z_{4} z_{5}-\left(\psi z_{1} z_{2} z_{3} z_{4} z_{5}\right)^{2}>
\end{gathered}
$$

Obviously, the r.h.s. can be reproduced by moduli-derivatives. After some arithmetics we obtain the Picard-Fuchs equation in the form:

$$
\left(\mathcal{D}_{1}^{2}-\left(\mathcal{D}_{2}+\frac{1}{6}\right)^{2}\right) \Pi=0, \quad \text { or } \quad\left(\mathcal{D}_{1}^{2}-\mathcal{D}_{2}^{2}\right)(\psi \Pi)=0
$$

\footnotetext{
15 As usual, Picard-Fuchs equations are for cohomology classes and do not depend on integration hypersurfaces. For the same reason we do not need to write $i=\sqrt{-1}$-factors in the exponents in $(9.3)$ and $(9.4)$ - they affect only the integration paths and parametrization of moduli. Of course only for appropriate integration domains the Ward identites which we use can be valid. Note also that our notation is slightly different from [13]: our $\phi=-\phi_{\mathrm{KKLMV}}$ and our $\psi=-(864 i)^{1 / 6} \psi_{\mathrm{KKLMV}}$ so that parameters $X=\frac{\phi}{i \psi^{6}}, Y=\frac{1}{\phi^{2}}, X_{1}=\frac{1}{\left(\phi-i \psi^{6}\right)^{2}}, X_{2}=\frac{\phi-i \psi^{6}}{\psi^{6}}=-i\left(1-\frac{\phi}{i \psi^{6}}\right)$.
} 
where operators $\mathcal{D}_{12}$ are given by ${ }^{16}$

$$
\mathcal{D}_{1}=\frac{\partial}{\partial \phi}, \quad \mathcal{D}_{2}=\phi \frac{\partial}{\partial \phi}+\frac{1}{6} \psi \frac{\partial}{\partial \psi} .
$$

Note, that this equation is essentially insensitive to the part of defining polynomial $p(z \mid \phi, \psi)$ which depends on $z_{3}, z_{4}$ and $z_{5}$ : it only matters that they all interact with $z_{1}$ and $z_{2}$ through the linear term $\psi z_{1} z_{2} f\left(z_{3}, z_{4}, z_{5}\right)$ and the coefficient $\psi$ is a modulus. Of course, as in the $S L(2)$ Calogero case, since we are now in the situation with two moduli, the second Picard-Fuchs equation is also needed. But it should be higher order in derivatives and does not add to our discussion.

\subsection{The double scaling limit of Picard-Fuchs equation}

As we already know, in this limit $\phi$ and $\psi \rightarrow \infty$, while $\tilde{u} \equiv \frac{u}{\Lambda^{2}}=\phi-i \psi^{6}$ remains finite. Moreover,

$$
\tilde{\Pi}=\psi^{-4} \Pi,
$$

this can be shown either by saddle-point evaluation of $z_{3,4,5}$-integrals in (9.3) - what gives (9.4) with an extra factor of $\psi^{-4}$, or by comparison of the equation that we obtain shortly with (7.12). For such $\tilde{\Pi}$ the Picard-Fuchs equation (9.11) becomes:

$$
\left(\mathcal{D}_{1}^{2}-\left(\mathcal{D}_{2}-\frac{1}{2}\right)^{2}\right) \tilde{\Pi}=0, \quad \text { or } \quad\left(\mathcal{D}_{1}-\mathcal{D}_{2}\right)\left(\mathcal{D}_{1}+\mathcal{D}_{2}-1\right) \tilde{\Pi}=\frac{1}{4} \tilde{\Pi} .
$$

Perform now a change of variables: $\{\phi, \psi\} \rightarrow\{\phi, u\}$. Then,

$$
\left.\frac{\partial}{\partial \phi}\right|_{\psi=\text { const }}=\left.\frac{\partial}{\partial \phi}\right|_{u=\text { const }}+\Lambda^{2} \frac{\partial}{\partial u}, \quad \frac{\partial}{\partial \psi}=-6 i \Lambda^{2} \frac{\partial}{\partial u} .
$$

After such substitution (9.14) turns into:

$$
\left(\frac{\partial}{\partial \phi}+\Lambda^{2} \frac{\partial}{\partial u}\right)^{2} \tilde{\Pi}=\left(\phi \frac{\partial}{\partial \phi}+u \frac{\partial}{\partial u}-\frac{1}{2}\right)^{2} \tilde{\Pi},
$$

and assuming $\frac{\partial \tilde{\Pi}}{\partial \phi}=0$ for the leading term in the $\frac{1}{\phi}$ expansion of $\tilde{\Pi}$, we obtain $(7.12)$, or, what is the same, (9.8):

$$
\left(\left(u^{2}-\Lambda^{2}\right) \frac{\partial^{2}}{\partial u^{2}}+\frac{1}{4}\right) \tilde{\Pi}=0 .
$$

\footnotetext{
${ }^{16}$ Remarkably, the algebra formed by these operators, $\left[\mathcal{D}_{1}, \mathcal{D}_{2}\right]=\mathcal{D}_{1}$, has another well known representation: in terms of $p$ and $q$, satisfying $[p, q]=1: \hat{\mathcal{D}}_{1}=e^{q}, \hat{\mathcal{D}}_{2}=p$ - so that (9.11) becomes the Shroedinger equation for $1 d$ Liouville model: $\left(p^{2}-e^{2 q}\right) \Pi=0$. This important analogy is, however, beyond the scope of this paper. For the relevant theory of Liouville wave functions see [32] and references therein.
} 


\subsection{Comments}

Equation (9.11) is similar enough to eq.(8.43) for $1 d$ Calogero model to confirm the belief that the two are closely related, though the equations are clearly not the same (this is especially obvious if Calogero-case equation is convertable into something like (8.65)). The most striking difference is appearence of two second-order differential operators in (9.11), while one of them is of the first order in (8.43). It deserves emphasizing that this first order derivative is exactly what makes (8.65) look like a Schroedinger equation - and thus it is not very easy to give up. This discreapancy - if not eliminated by a clever change of variables,- deserves explanation. If eliminated (and if the second Picard-Fuchs equations are also compatible), it would strongly suggest that the stringy Calabi-Yau and $1 d$ Calogero belong to the same Whitham universality class - not a big surprise, because the Whitham theory classifies models according to the number of their moduli: extreme low-energy degrees of freedom rather than to that of ordinary degrees of freedom at high energies and models which are very different in the UV can become the same in the IR. Most probably, however, the difference between (9.11) and (8.43) is real, and disappears only in the limit $\alpha^{\prime} \rightarrow 0$. It is very appealing to think that (9.11) is a "relativistic" version of (8.43) - when the linear "time"-derivative turns into the second-order one. If correct, this would strongly imply that the Ruijsenaars model the "relativistic" version of Calogero system - can adequately describe (9.11). For our purpose this model is just the same as Calogero one, just the group $G(G=S L(2)$ in most of our examples) is deformed to become quantum group, $G_{s}=S L_{s}(2)$, with the "Planck-constant" $\log s \sim \alpha$. Unfortunately, our prelimiary analysis in s.10 below does not fully confirm these expectations. It can be, however, too naive in its present form.

Another remark: there are 6 periods on the Calabi-Yau manifold in question, and only two of them can be related to $a$ and $a^{D}$ of Calogero model. The others are similarly related to the

"trivial" periods $(1, \tau)$ of $E(\tau)$, which are neglected when $\mathcal{C}$ is substituted by $\hat{\mathcal{C}}$, see eq. (8.8). This statement is in agreement with the results of [13]: the other 4 periods in the double scaling limit are $(1, S, u, u S)$, and in this limit $S \sim \tau$.

\section{Ruijsenaars model: beginning of the story}

Since this model have not been analyzed in the context of Seiberg-Witten theory in [4], we need several extra formulas to begin with. The Lax matrix for Ruijsennars model - which we need to 
define the full spectral curve $\mathcal{C}$ from eq.(6.1) - is [29]:

$$
L_{i j}(\xi)=e^{P_{i}} \frac{F\left(q_{i j} \mid \xi\right)}{F\left(q_{i j} \mid \mu\right)} \prod_{l \neq i} n(\mu) \sqrt{\wp(\mu)-\wp\left(q_{i l}\right)},
$$

where $i, j, l=1, \ldots, N_{c}, \wp(\xi)=-\frac{\partial \zeta(\xi)}{\partial \xi}=-\frac{\partial^{2} \log \sigma(\xi)}{\partial \xi^{2}}$

$$
F\left(q_{i j} \mid \xi\right)=\frac{\sigma\left(q_{i}-q_{j}+\xi\right)}{\sigma(\xi) \sigma\left(q_{i}-q_{j}\right)},
$$

and normalization constant $n(\mu)$ can be fixed as convenient, most often it is taken to be $n(\mu)=\sigma(\mu)$ - this simplifies the formulas in the Calogero limit $\mu \rightarrow 0$. The bare spectral curve - to which the spectral parameter $\xi$ belongs in eq.(10.1) is our usual elliptic $E(\tau)$.

$L_{i j}(\xi)$ in (10.1) depends on an extra parameter $\mu$, as compared to Calogero model, and Calogero Lax operator [30] is recovered in the limit $\mu \rightarrow 0, P_{i}=\frac{\mu}{g} p_{i}+o\left(\mu^{2}\right)[29]$ :

$$
L_{i j}(\xi)=\delta_{i j}+\frac{\mu}{g}\left(p_{i} \delta_{i j}+\left(1-\delta_{i j}\right) g F\left(q_{i j} \mid \xi\right)\right)+o\left(\mu^{2}\right) .
$$

The spectral curve $\mathcal{C}$ for the Ruijsenaars model, as defined in (6.1), is:

$$
\mathcal{C}: \quad \operatorname{det}\left(t \delta_{i j}-L_{i j}(\xi)\right)=0,
$$

and according to (6.2),

$$
d \mathcal{S}_{\min }^{R u}=2 t d \omega_{0}(\xi)
$$

We now restrict ourselves to our usual example of $G=S L(2)$, i.e. $N_{c}=2$. Then (10.3) is

$$
t^{2}-t \operatorname{trL}+\operatorname{det} L=0
$$

and, denoting $P \equiv P_{1}=-P_{2}, q \equiv q_{1}-q_{2}$,

$$
\begin{gathered}
H \equiv \frac{1}{2 n(\mu)} \operatorname{tr} L=\frac{1}{2}\left(e^{P}+e^{-P}\right) \sqrt{\wp(\mu)-\wp(q)}, \\
\operatorname{det} L=n^{2}(\mu)(\wp(\mu)-\wp(\xi)),
\end{gathered}
$$

(to get these formulas one can use the identity $F(q \mid \xi) F(-q \mid \xi)=\wp(q)-\wp(\xi)$, see [4] for generalization to higher- $N_{c}$ case). $H=H(\mu)$ that appeared here is the single independent integral of motion of the $S L(2)$-Ruijsenaars model. In terms of it, we rewrite (10.3) as:

$$
t=\frac{H \pm \sqrt{H^{2}-\wp(\mu)+\wp(\xi)}}{n(\mu)}
$$

and

$$
n(\mu) d \mathcal{S}_{\min }^{R u}=2 H d \omega_{0} \pm\left. d \mathcal{S}_{\min }^{C a l}\right|_{\hat{h}=H^{2}-\wp(\mu)}
$$


where $d \mathcal{S}_{\min }^{C a l}=2 \sqrt{\hat{h}+\wp(\xi)} d \omega_{0} \sim \frac{\sqrt{\hat{h}-x}}{y(x)} d x$, with $\hat{h}=H^{2}(\mu)-\wp(\mu)$.

We see immediately that the substitution (projection) $\mathcal{C} \rightarrow \hat{\mathcal{C}}(8.8)$, that played a certain role in our analysis of Calogero model, is a little more tricky in the context of Ruijsenaars model. Actually, it is only $d \mathcal{S} \equiv n(\mu) d \mathcal{S}_{\min }^{R u}-2 H d \omega_{0}$ that can be projected on $\hat{\mathcal{C}}$ without loss of information about the periods. Instead, the piece $2 H d \omega_{0}$ keeps track of the periods of $E(\tau)$ - which are completely ignored by $d \mathcal{S}_{\min }^{C a l}$. This is in accordance with one of our expectations in s.9.5 - that more periods can be revealed in the Ruijsenaars model context - as required to make things consistent with Calabi-Yau picture.

However, the Picard-Fuchs equation does not change as much as necessary. Essentially, $d \mathcal{S}$ satisfies just the same equation (8.43): the only new thing is that one should subtract $2 H d \omega_{0}$ and change the variables. Eq.(8.43) is written in variables $(\hat{h}, \tau)$, while now we rather need $(H, \tau)$. Since

$$
\left.\frac{\partial}{\partial \tau}\right|_{H}=\left.\frac{\partial}{\partial \tau}\right|_{\hat{h}}+\frac{\partial \hat{h}}{\partial H} \frac{\partial}{\partial \hat{h}}, \quad \frac{\partial}{\partial H}=\frac{\partial \hat{h}}{\partial H} \frac{\partial}{\partial \hat{h}}
$$

this change of variables does not seem to produce second-order $\tau$-derivatives and thus can hardly eliminate the main discrepancy with the Calabi-Yau-case equation (9.11).

Still, this analysis of the Ruijsenaars model is preliminary and can appear oversimplified. In any case the problem deserves attention and requires more studies in the future.

\section{Open questions}

Before concluding this paper we briefly list the problems that did not allow us to make the presentation completely closed.

As concerns the abstract theory of the prepotential, discussed in the first half of the paper,

- Our presentation was essentially based on the use of the "quasiclassical Baker-Akhiezer function" $d S$ (or $\Omega$ in sec.5). Quasiclassical $\tau$-function (of which the prepotential is a logarithm) appears as a secondary object. The proper presentation should be inverse: the quasiclassical $\tau$ function should be introduced first, and Baker-Akhiezer function emerge from it. In the theory of ordinary $\tau$-functions these are defined as generating functionals of all the matrix elements of a universal group element of some Lie algebra, and - as such - satisfy the (generalized) bilinear Hirota equations (which are nothing but comultiplication formula, see [31] and references therein). In the particular case of level-one affine (Kac-Moody) algebras - when conventional (multicomponent) KP/Toda hierarchies arise - one can proceed further: define the Baker-Akhiezer function by eq.(4.13) and it will be a function on (section over) a complex curve. Even for the ordinary 
$\tau$-functions the substitute of Miwa transform and thus of eq.(4.13) in the general situation (for arbitrary Lie groups, not obligatory level-one and 1-loop) is unknown. It is suggested in [6] that analogous abstract definition of quasiclassical $\tau$-functions should be based on the concept of Frobenius, rather than Lie algebras - but these one are much less understood, to say the least. In any case, emergence of $d \mathcal{S}$ - and algebraic criteria of when it arises as living on the spectral curves and when on some higher-dimensional manifolds (i.e. when one should use $\Omega$ from sec.5 above instead of $d \mathcal{S})$ - in such context remain unclear.

- Relation between group theory (ordinary $\tau$-functions) and Hodge structures (quasiclassical ones) - which in physical language should be relation between the entire model and its topological (low-energy) limit - is still obscure. Of course it is well understood in the particular context of Whitham limit of $\mathrm{KP} /$ Toda $\tau$-function (see [7] and references therein), but again, in general abstract terms things are not enough clarified.

- Conceptual relation between the prepotential, considered as a full generating function (i.e. with infinitely many "time"-variavles), and the reduced one, defined with the help of particular $d \mathcal{S}$ or $\Omega$ should be understood better: this is just another formulation of the previous problems.

- Even in the setting to which we had to restrict our presentation, there remains a small subtlety: divergence of the prepotential in the presence of simple poles in $d \mathcal{S}$ and $\Omega$ (in the Seiberg-Witten theory these arise whenever there are massive matter multiplets). Though not very harmfull as explained in the main text - regularization of these divergencies is an additional step in the construction (which can also introduce extra moduli, characterizing the choice of regularization), which should be made somewhat less artificial than it is now.

As concerns the second part of the paper: examples of how the general theory can be applied to particular Seiberg-Witten/integrable models, we mostly concentrated on the $S L(2)$ case. Thus,

- Generalization to other groups, most interestingly to quantum and affine groups, is not discussed. It is rather straightforward, though sometime technically tedious, especially in the case of Calabi-Yau manifolds. Adequate technical means should be developed to handle generic situation, especially the Picard-Fuchs equations and Calogero $(N=2 \rightarrow N=4$ flow $)$ case.

- A separate issue is adequate treatement of models with matter multiplets in the fundamental of the gauge group. This is not a really big problem at the first step, because it is enough to allow more punctures on the bare spectral curve in order to get some description, but the next steps - like embedding of the model into an UV-finite one (say, the substitute of Calogero-model description for the $N_{f}=4$ model) - can hardly be made without adequate techniques. 
Finally, even for the $S L(2)$ case we did not fully resolve three kinds of problems:

- We did not treat the modulus $\tau$ in Calogero system on equal footing with the other arguments of the prepotential: there is no "symmetry" between $\hat{h}$ and $\tau$.

- A full understanding of Picard-Fuchs equation (8.43) in Calogero case is not achieved: see ss.8.8 and 8.9 for details.

- Relation between Calabi-Yau and Calogero models is not completely revealed, see discussion in s.9.5. Once found it could be an important step towards classification of prepotentials in the simplest non-trivial case of two moduli.

To this list of "technical" problems one should of course add a string of conceptual ones from [3]: At one end would be understanding of Whitham theory as a general method for description universality classes of renormalization-group flows and - at the same time - of quantization of the field theory. At another end would be classification of all possible Witham universality classes possible if the theory is formulated in abstract terms. When worked out and then combined the answers to these conceptual questions will definitely have interesting applications.

\section{Conclusion}

To conclude, we presented a preliminary discussion of quasiclassical $\tau$-functions, or prepotentials - the old-known objects, which nowadays acquire the role they deserve in theoretical physics. As ordinary (generalized) $\tau$-functions are supposed to describe generic effective actions in the space of coupling constants and exhibit their intrinsic group-theoretical nature, the quasiclassical ones should play the same role at the end-point of renormalization-group evolution: in the IR limit and the corresponding theory should reveal the hidden dynamical symmetry of effective models on the moduli spaces. Unfortunately, this theory at the present stage is not so clear as in the case of the usual $\tau$-functions: it more resembles the "phenomenology" of $\tau$-functions before they were identified with universal group elements. By now standard identification of the prepotential (Whitham) theory with that of the Hodge structures - which is used in the present paper - is at best the first step towards the general theory. As a necessary minimum, one should clearly explain, why (and in what sense) the Hodge structures always appear at the IR stable points of renormalization evolution of group theory (by now it is a problem even to define what these words could mean in the absolutely general setting).

Still, even with the present state of knowledge, the theory has applications - of which we briefly discussed the most fresh and exciting ones: to Seiberg-Witten description of $N=2$ SUSY gauge 
models and its lifting to Calabi-Yau models (the yet-remembered previous avatara was in topological field theory). We paid some attention to the homogeneity property, peculiar to all prepotentials - which reflects its invariance under the simplest constituent of renormalization-group evolution (in)dependence on the overall scale. As soon as some moduli are fixed, the reduced prepotential acquires anomalous dimension, defined in the Seiberg-Witten setting by its inclusion into anomaly multiplet, ${ }^{17}$ along with $T_{\mu \mu} \sim \beta_{W}<\operatorname{tr} G^{2}>$ and $\partial_{\mu} J_{\mu}^{5} \sim \beta_{W}<\operatorname{tr} G \tilde{G}>$ :

$$
2 \mathcal{F}-\sum_{i} a_{i} \frac{\partial \mathcal{F}}{\partial a_{i}} \sim \beta_{W}<\operatorname{tr} \phi^{2}>
$$

(all the relations acquire corrections when massive matter fields are introduced). This simple example illustrates the very spirit of the general theory (or rather entire "stringy" philosophy): as soon as all possible parameters (including IR and UV cut-offs, counterterms, bare couplings etc) are introduced in the game as either coupling constants or background fields, - the hidden symmetries are revealed, and they often do have implications for original reduced model.

Clearly, much more should be done, in order to appreciate the real significance of the string program for physics, as to the examples considered in these notes. They can hopefully add to the evidence that the Whitham theory is already a full-fledged constituent of the whole approach.

\section{Acknowledgements}

We benefited from discussions with O.Aharony, L.Alvarez-Gaume, S.Das, A.Gerasimov, C.Gomez, A.Gorsky, A.Hanany, A.Losev, I.Krichever, H.Kunitomo, A.Marshakov, A.Mironov, N.Nekrasov,

\footnotetext{
${ }^{17} \beta_{W}$ is Wilsonian $\beta$-function, which is always constant (one-loop) in supersymmetric gauge theories in the absence of massive matter fields. In one word, the field-theory derivation of (12.1) is just the same as the usual one for the trace anomaly: Consider effective action

$$
\exp \int \mathcal{F}\left(\Phi_{\mathrm{cl}}\right) d^{4} x d^{4} \theta=\int \mathcal{D} \Phi_{\mathrm{qu}} \exp \int F\left(\Phi_{\mathrm{cl}}+\Phi_{\mathrm{qu}}\right) d^{4} x d^{4} \theta \equiv<1>_{\Phi_{\mathrm{cl}}}
$$

and vary with respect to the scale $\Lambda$. Then, since the prepotential at the l.h.s. depends on $\Lambda$ only through $\phi_{\mathrm{cl}} / \Lambda$,

$$
\int\left(2 \mathcal{F}-\sum_{i} \Phi_{i} \frac{\partial \mathcal{F}}{\partial \Phi_{i}}\right) \delta \Lambda d^{4} x d^{4} \theta=\left\langle\left\langle\int \frac{\partial F}{\partial \Lambda} \delta \Lambda d^{4} x d^{4} \theta\right\rangle\right\rangle
$$

If one wants to recover the axial-current anomaly from the same derivation, one formally allows for complex variations $\delta \Lambda$. Similarly, allowing nilpotent $\delta \Lambda$ we obtain this relation for all the components of the superfield, not just for the highest one. Since the highest component of $\frac{\partial F}{\partial \Lambda}$ is $\beta_{W} \operatorname{tr} G^{2}$, the lowest one is obviously $\beta_{W} \operatorname{tr} \Phi^{2}$. Comparing this with the lowest component at the l.h.s. we get (12.1). More accurately, one should couple the theory to the $N=2$ supergravity and vary w.r.to rescaling of the gravitational supermultiplet. Also one should treat auxiliary fields more carefully (note that the bare prepotential $F$ is non-abelian and therefore can be described only with inclusion of infinitely many auxiliary superfields).
} 
K.Ohta, M.Olshanetsky, I.Polyubin, A.Rosly, V.Rubtsov, J.Sonnenschein and A.Tokura.

H.I. is supported in part by Grant-in-Aid for Scientific Research (07640403) from the Ministry of Education, Science and Culture, Japan. A.M. acknowledges the hospitality of Osaka University and support of the JSPS.

\section{References}

[1] N.Seiberg and E.Witten, Electric-Magnetic Duality, Monopole Condensation and Confinement in $N=2$ Supersymmetric Yang-Mills Theory, Nucl.Phys. B426 (1994) 19-52; Err.: ibid. B430 (1994) 485-486, hep-th/9407087.

[2] N.Seiberg and E.Witten, Monopoles, Duality and Chiral Symmetry Breaking in $N=2$ Supersymmetric QCD, ibid. B431 (1994) 484-550, hep-th/9408099.

[3] A.Gorsky, I.Krichever, A.Marshakov, A.Mironov et al., Integrability and Exact Seiberg-Witten Solution, Phys.Lett. 355B (1995) 466-474, hep-th/9505035.

[4] H.Itoyama and A.Morozov, Integrability and Seiberg-Witten Theory. Curves and Periods, preprint ITEP-M5/95/OU-HET-227, hep-th/9511126.

[5] I.Krichever, The $\tau$-function of the Universal Whitham Hierarchy, Matrix Models and Topological Field Theories, hep-th/9205110;

I.Krichever, The Dispersionless Lax Equations in Topological Minimal Models, Comm. Math.Phys. 143 (1992) 415-429.

[6] B.Dubrovin, Geometry of 2d Topological Field Theories, SISSA-89/94/FM;

B.Dubrovin, Integrable Systems in Topological Field Theory, Nucl.Phys. B379 (1992) 627-689; B.Dubrovin, Hamiltonian Formalism of Witham-type Hierarchies in Topological LandauGinzburg Model, Comm.Math.Phys. 145 (1992) 195.

[7] T.Nakatsu and K.Takasaki, Whitham-Toda Hierarchy and $N=2$ Supersymmetric Yang-Mills Theory, hep-th/9509162.

[8] T.Eguchi and S.K.Yang, Prepotentials of $N=2$ Supersymmetric Gauge Theories and Soliton Equations, hep-th/9510183.

[9] J.Sonnenschein, S.Theisen and S.Yankielowicz, On the Relation between the Holomorphic Prepotentials and the Quantum Moduli in SUSY Gauge Theories, hep-th/9510129. 
[10] See, for example,

A.Morozov, String Theory, what is it, Rus.Physics Uspekhi 35 (1992) 671 (v.162, No.8, p.84175 of Russian edition);

A.Morozov, Integrability and Matrix Models, ibid. 164 (1994) No.1, 3-62 (Rus.ed.), hepth/9303139.

[11] I.Krichever, Methods of Algebraic Geometry in the Theory of Nonlinear Equations, Sov.Math.Surveys, 32 (1977) 185-213;

I.Krichever, The Integration of Non-linear Equations by the Methods of Algebraic Geometry, Funk.Anal. and Appl. 11 (1977) No.1 15-31 (Rus.ed.);

I.Krichever, Elliptic Solutions of the Kadomtsev-Petviashvili Equation and Integrable System of Particles, Funk.Anal. and Appl. 14 (1980) 282-290 (No.4 15-31 of Rus.ed.);

B.Dubrovin, Theta Functions and Non-linear Equations, Sov.Math.Surveys, 36 (1981) No.2 11-80 (Rus.ed.)

[12] A.Strominger, Massless Black Holes and Conifolds in String Theory, Nucl.Phys. B451 (1995) 96, hep/th 9504090 .

[13] S.Kachru, A.Klemm, W.Lerche, P.Mayr and C.Vafa, Nonperturbative Results on the PointParticle Limit of $N=2$ Heterotic String Compactification, hep/th 9508155.

[14] E.Martinec and N.Warner, Integrability in $N=2$ Gauge Theory: A Proof, hep-th/9511052.

[15] A.Klemm, W.Lerche and S.Theisen, Nonperturbative Effective Actions of $N=2$ Supersymmetric Gauge Theories, hep-th/9505150;

M.Matone, Instantons and Recursion Relation in $N=2$ Gauge Theory, hep-th/9506102;

M.Matone, Koebe 1/4-Theorem and Inequalities in $N=2$ Super-QCD, hep-th/9506181;

K.Ito and S.-K.Yang, Prepotentials in $N=2 S U(2)$ Supersymmetric Yang-Mills Theory with Massless Hypermultiplets, hep-th/9507144.

[16] B.de Wit, P.Lanwers and A.van Proeyen, Lagrangians of $N=2$ Supergravity-Matter Systems, Nucl.Phys.B255 (1985) 269;

A.Strominger, Special Geometry, Comm.Math.Phys. 133 (1990) 163;

A.Ceresole, R.D'Auria and S.Ferrara, On the Geometry of the Moduli Space of Vacua in $N=2$ Supersymmetric Yang-Mills Theory, Phys.Lett. 339B (1994) 71, hep-th/9408036;

and references therein.

[17] E.Martinec and N.Warner, Integrable Systems and Supersymmetric Gauge Theories, hepth/9509161. 
[18] R.Donagi and E.Witten, Supersymmetric Yang-Mills Theory and Integrable Systems, IASSNSHEP-95-78, hep-th/9510101.

[19] E.Martinec, Integrable Structures in Supersymmetric Gauge and String Theory, hepth/9510204.

[20] A.Gorsky and A.Marshakov, Towards Effective Topological Gauge Theories on Spectral Curves, ITEP/TH-9/95, hep-th/9510224.

[21] C.Gomez, R.Hernandez and E.Lopez, $S$-Duality and the Calabi-Yau Interpretation of the $N=4$ to $N=2$ Flow, hep-th/9512017.

[22] A.Klemm, W.Lerche, S.Theisen and S.Yankielowicz, Simple Singularities and $N=2$ Supersymmetric Yang-Mills Theory, Phys.Lett. 344B (1995) 169, hep-th/9411048.

[23] P.Agyres and A.Faraggi, The Vacuum Structure and Spectrum of $N=2$ Supersymmetric SU(N) Gauge Theory, Phys.Rev.Lett. 73 (1995) 3931, hep-th/9411057.

[24] A.Hanany and Y.Oz, On the Quantum Moduli Space of Vacua of $N=2$ Supersymmetric $S U\left(N_{c}\right)$ Gauge Theories, hep-th/9505075.

[25] For all the relevant details about hyperelliptic curves see, for example, V.Knizhnik, Analytic Fields on Riemann Surfaces, Comm.Math.Phys. 112 (1987) 567;

D.Lebedev et al., Statistical Sums of Strings on Hyperellyptic surfaces, Nucl.Phys.B302 (1988) 163-188;

V.Knizhnik, Multiloop Amplitudes in the Theory of Quantum Strings and Complex Geometry, Sov.Physics Uspekhi, 159 (1989) No.3 401-453 (Russian edition).

[26] V.Inosemtsev, The Finite Toda Lattices, Comm.Math.Phys. 121 (1989) 629-638.

[27] M.Olshanetsky Generalized Hitchin Systems and the Knizhnik-Zamolodchikov-Bernard Equation on Elliptic Curves, hep-th/9510143.

[28] S.Kachru and C.Vafa, Exact Results for $N=2$ Compactifications of Heterotic Strings, hepth/9505105;

and references therein.

[29] S.Ruijsenaars, Complete Integrability of Relativistic Calogero-Moser System and Elliptic Function Identities, Comm.Math.Phys. 110 (1987) 191-213. 
[30] I.Krichever, Elliptic Solutions of the Kadomtsev-Petviashvili Equation and Integrable System of Particles, Funk.Anal. and Appl. 14 (1980) 282-290 (No.4 15-31 of Rus.ed.)

[31] A.Gerasimov, S.Khoroshkin, D.Lebedev, A.Mironov et al., Generalized Hirota Equations and Representation Theory. The Case of $S L(2)$ and $S L_{2}(q)$, Mod.Phys.Lett.A10 (1995) 2589-2614, hep-th/9405011.

[32] A.Gerasimov, S.Kharchev, A.Marshakov, A.Mironov, M.Olshanetsky et al, Liouville Models in Group Theory Framework, preprint ITEP-M4/95. 